\title{
Spirocyclic tetramates by sequential Knoevenagel and [1,5]-prototropic shift
}

\author{
Laia Josa-Culleré, ${ }^{\ddagger}$ Michael G. Hirst, ${ }^{\ddagger}$ Jonathan P. Lockett, ${ }^{\star}$ Amber L. Thompson ${ }^{\star}$ and Mark G. Moloney ${ }^{* \dagger \dagger}$ \\ ${ }^{\ddagger}$ Chemistry Research Laboratory, University of Oxford, Mansfield Rd, University of Oxford, OX1 3TA, U.K and \\ †xford Suzhou Centre for Advanced Research, Building A, 388 Ruo Shui Road, Suzhou Industrial Park, Jiangsu, 215123, P.R. China.
}

Keywords: Spirocycles; Tetramates; Antibacterial

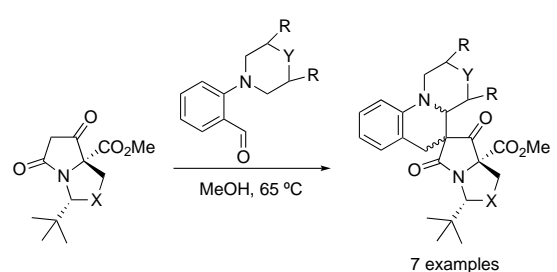

Highly functionalised spirocyclic tetramates were prepared via a sequential Knoevenagel reaction and [1,5]-prototropic shift (Treaction) of bicyclic tetramates. While these compounds isomerise in solution, stable analogues can be prepared via appropriate choice of substituents. Further modification of these compounds allows for introduction of aromatic groups, making them suitable as skeletons suitable for application in medicinal chemistry.

\section{Introduction}

It is now widely appreciated that the antibacterial drug pipeline is poorly populated, ${ }^{1-3}$ that models for antibacterial drug development need revision, ${ }^{4-8}$ and that the emergence of antibacterial resistance creates a constant need for new drugs. ${ }^{9-11}$ Therefore, the identification of suitable and effective drug discovery paradigms has become urgent. $^{12-18}$ Recently, spiropyrimidinetrione PNU-286607 1 (Figure 1) was reported to have a broad spectrum antibacterial activity, including against fluoroquinoline resistant strains, and a novel mode of action. ${ }^{19} \mathrm{It}$ was shown to target the $\beta$ subunit of bacterial type II topoisomerases via a novel mechanism of inhibition. The common pharmacophore of the class has been extended to include $2{ }^{20,21} \mathbf{3}^{22}$ and AZD0914 $4^{23}$ (Figure 1). The (-)-enantiomer exhibits both the highest antibacterial activity along with the highest inhibitory effects, while the (+)-isomer is inactive. More recently, 4 has also shown high activity against the multidrug-resistant Neisseria gonorrhoeae, giving it the potential to combat the growing public health concern of gonorrhea. ${ }^{24,25}$
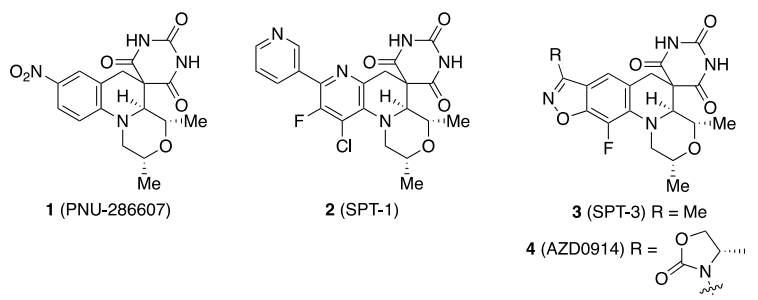

Figure 1. Examples of biologically active spiropyrimidinetriones.

The synthesis of these systems relies on a very effective intramolecular cyclisation, known as the "T-reaction" or the " $t$ amino effect", 26 which involves a suprafacial [1,5]-prototropic shift followed by carbon-carbon bond formation (Scheme 1). The transformation is promoted when a tertiary aromatic amine is substituted with an ortho-electron-poor double bond, and it has been successfully employed to access a range of tricyclic quinoline systems with multiple stereocentres. ${ }^{27-30}$ The asymmetric synthesis of 1 has been developed by Hurd and co-workers, ${ }^{31}$ where transdimethylmorpholine was used as a source of asymmetry, and the reaction was driven towards the thermodynamic product where the substituents in the morpholine ring were equatorial. This proceeded via isomerisation of the initial kinetic intermediate. While the barbiturate nucleus is well suited to this process, ${ }^{31-33}$ given our interest in related tetramates both for antibacterial drug discovery ${ }^{34}$ and for the generation of novel 3-D drug templates, ${ }^{35}$ we examined them for their suitability in the T-reaction. Access to spirocyclic tetramates has been rarely reported. ${ }^{36-39}$ In our case, an additional level complexity is added by the chiral nature of the tetramate, as opposed to the non-chiral barbiturate, which converts the spirocentre into yet another quaternary carbon.

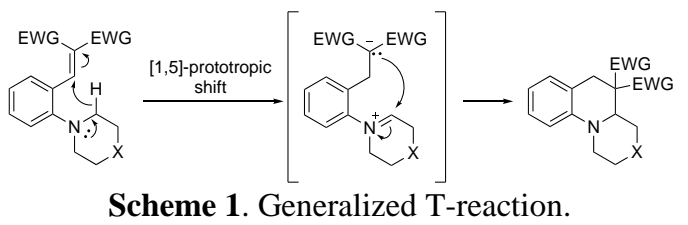

\section{Results and Discussion}

\subsection{Synthesis of spirocyclic tetramates}

The synthesis of the required bicyclic tetramate cores 5a,b (Schemes 2 and 3), which are readily available from serine and cysteine, has been reported. ${ }^{40}$ The required $o$-aminobenzaldehydes 6a-d were obtained by reaction of cyclic amines with $o$ fluorobenzaldehyde using literature methodology (Scheme 3). ${ }^{41}$
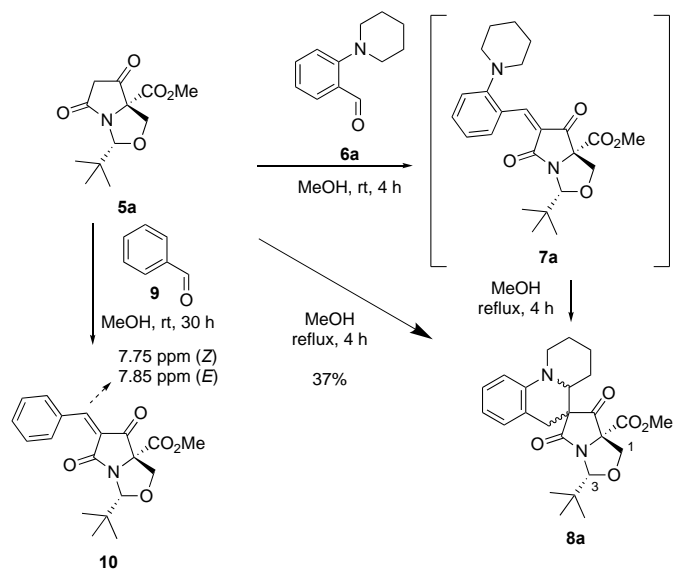

Scheme 2. Synthesis of spirocyclic tetramate $\mathbf{8 a}$ and alkylidene $\mathbf{1 0 .}$

In the first attempt to access the spirocyclic tetramate, it was found that upon mixing tetramate $\mathbf{5 a}$ with aldehyde $\mathbf{6 a}$ in methanol at reflux and subsequent cooling, spontaneous 
precipitation took place to afford spiro-tetramate 8a (Scheme 2 ). The isolated product was found to be a mixture of diastereoisomers, which were quantified by the $H(1)$ doublets and the $H(3)$ singlet. Interestingly, the number of isomers and the diastereomeric ratio changed depending on the solvent used (Table 1). While non-polar solvents $\left(\mathrm{C}_{6} \mathrm{D}_{6}\right.$, tol- $\left.\mathrm{d}_{8}\right)$ gave a single isomer (minor isomer <2\%), with DMSO and acetone 3 isomers were observed. These data suggest that 8a obtained as a precipitate is a single isomer, but that it may isomerise upon dissolution.

Table 1. Solvent dependence of the number of isomers of $\mathbf{8 a}$. Spectra recorded 5-10 min after dissolving the precipitate.

\begin{tabular}{|c|c|c|c|c|c|c|}
\hline Solvent & $\begin{array}{l}\text { DMSO- } \\
d_{6}\end{array}$ & $\begin{array}{l}\text { Acetone- } \\
d_{6}\end{array}$ & $\mathrm{CDCl}_{3}$ & DCM-d ${ }_{2}$ & Tol-d $_{8}$ & $\mathrm{C}_{6} \mathrm{D}_{6}$ \\
\hline $\begin{array}{l}\text { No. } \\
\text { isomers }\end{array}$ & 3 & 3 & 2 & 2 & 1 & 1 \\
\hline $\begin{array}{l}H(3) \mathrm{NM} \\
\text { peaks }^{*}\end{array}$ & & & & & & \\
\hline d.r. & $3: 6: 1$ & $5: 5: 1$ & $4: 6$ & & - & - \\
\hline
\end{tabular}

*Only 4.8-5.2 ppm shown

In an attempt to isolate the presumed alkylidene intermediate 7a, $5 \mathbf{a}$ and $6 \mathbf{a}$ were stirred at room temperature in methanol, but purification of 7a proved challenging as it cyclised to 8a both during flash column chromatography and in chloroform- $d$. That formation of $\mathbf{7 a}$, which would be expected from initial Knoevenagel condensation, was feasible, was shown by reaction of tetramate $\mathbf{5 a}$ with benzaldehyde $\mathbf{9}$. Under the same conditions, the alkylidene product $\mathbf{1 0}$ was isolated as a 1.2:1 mixture of the $E$ and $Z$ isomers (Scheme 2). The characteristic vinylic protons (7.75 - $7.85 \mathrm{ppm}$ ) of product $\mathbf{1 0}$ were not present in crude $\mathbf{8 a}$, which supports that the multiple products observed by NMR correspond to isomers of the spirocycle, and do not include intermediate 7a.

Treatment of tetramates $\mathbf{5 a , b}$ with a range of amines $\mathbf{6 a - d}$ (prepared by the reaction of $o$-fluorobenzaldehyde with amines 12a-d) following the same procedure (reflux in $\mathrm{MeOH}$ followed by precipitation and filtration) furnished spirocyclic tetramates 8ae in 7-58\% yield (Scheme 3 and Table 2). The morpholine analogues 8c-e were isolated at lower yields than that aliphatic derivatives $\mathbf{8 a}, \mathbf{b}$.

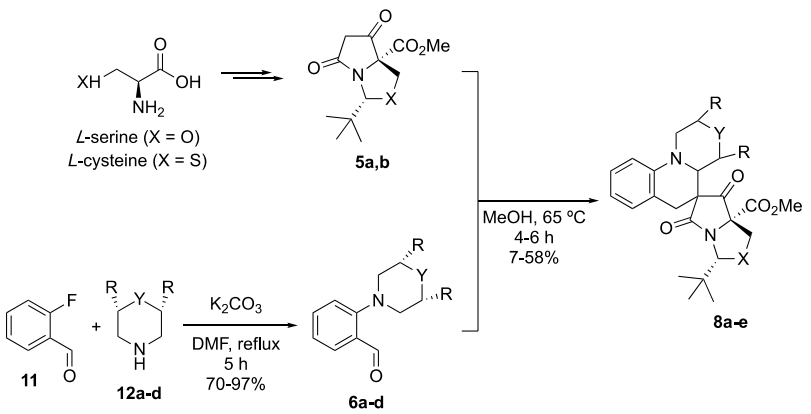

Scheme 3. Synthesis of aromatic amines 6a-d and spirocyclic tetramates 8a-e.

Examination of the cyclisation under alternative literature procedures was made, but refluxing in either ${ }^{\mathrm{n}}$ butanol at
$117{ }^{\circ} \mathrm{C}^{31}$ or in EtOH/ $\mathrm{H}_{2} \mathrm{O},{ }^{42}$ or adding pyrrolidine as a catalyst, ${ }^{43}$ resulted in either lower yield or no isolated product.

Table 2. Synthesis of spirocyclic tetramates 8a-e.

\begin{tabular}{|ccccccc|}
\hline Compound & $\mathbf{X}$ & $\mathbf{Y}$ & $\mathbf{R}$ & $\mathbf{Y i e l d}$ & $\begin{array}{c}\text { \# isomers } \\
\left(\mathbf{C}_{\mathbf{6}} \mathbf{D}_{\mathbf{6}}\right)\end{array}$ & d.r. \\
\hline $\mathbf{8 a}$ & $\mathrm{O}$ & $\mathrm{CH}_{2}$ & $\mathrm{H}$ & 37 & 2 & $98: 2$ \\
\hline $\mathbf{8 b}$ & $\mathrm{O}$ & - & $\mathrm{H}$ & 58 & 1 & - \\
\hline $\mathbf{8 c}$ & $\mathrm{O}$ & $\mathrm{O}$ & $\mathrm{H}$ & 7 & 1 & - \\
\hline $\mathbf{8 d}$ & $\mathrm{O}$ & $\mathrm{O}$ & $\mathrm{CH}_{3}$ & 31 & 2 & $8: 2$ \\
\hline $\mathbf{8 e}$ & $\mathrm{S}$ & $\mathrm{O}$ & $\mathrm{CH}_{3}$ & 23 & 2 & $8: 2$ \\
\hline
\end{tabular}

\subsection{Investigation into isomerism}

To investigate the stability of spirocycles 8a-e in solution, the isolated precipitates where dissolved in $\mathrm{C}_{6} \mathrm{D}_{6}$, and NMR spectra were recorded over time (Figure 2). While all analogues had equilibrated to 4 diastereomers after 2 weeks, the rate of isomerisation and ratio of isomers was dependent on the nature of the azacycle. The fastest rates were observed for piperidine $\mathbf{8 a}$, which reached equilibrium after $24 \mathrm{~h}$, and dimethyl-morpholine analogues $8 \mathbf{d}$ and $\mathbf{8 e}$, where equilibration was reached after 3 days. For pyrrolidine $\mathbf{8 b}$ and morpholine $\mathbf{8 c}$, new isomers were only observed after several days. Separation of the different isomers proved impossible, as the individual isomers had the same retention factors.

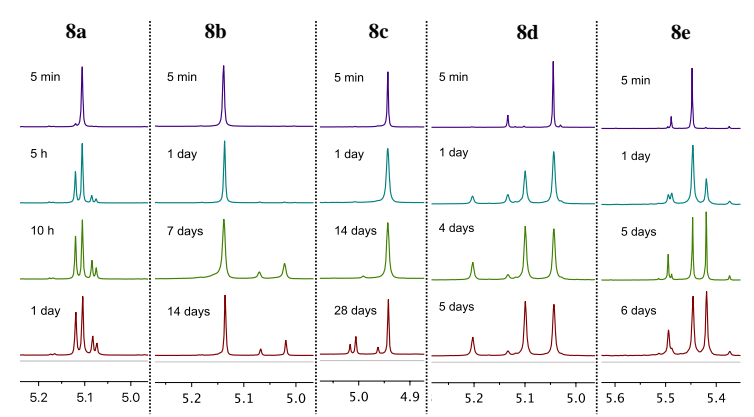

Figure 2. ${ }^{1} \mathrm{H}$ NMR spectra (in $\mathrm{C}_{6} \mathrm{D}_{6}$ ) of $H(3)$ singlet of spirocyclic tetramates 8a-e over time.

Isomerisation of these compounds probably involves an intramolecular mechanism where the spirocycle reverts to zwitterion 13 via a retro-Mannich type process, as indicated in Scheme 4. Such a phenomenon has been previously reported for related systems and has been utilised to access PNU-286607. ${ }^{21,31}$ It should be noted that in that case, epimerisation also occurred at the morpholine methyl substituent $\left(\left(C\left(4^{\prime}\right)-\mathrm{Me}\right)\right.$. For the spirocycles described herein, the formation of only 4 isomers for dimethyl-morpholine analogues 8d-e, which is the same number as for the unsubstituted 8a-c, suggests that no epimerisation at $C\left(4^{\prime}\right)$ occurs and that only the 2 stereocentres $C\left(4 \mathrm{a}^{\prime}\right)$ and $C(6)$ epimerise. Importantly, Ruble et al. observed epimerisation only when the mixture was heated at temperatures over $80{ }^{\circ} \mathrm{C},{ }^{31}$ and in our synthesis, spirocyclisation was performed at $65^{\circ} \mathrm{C}$. 


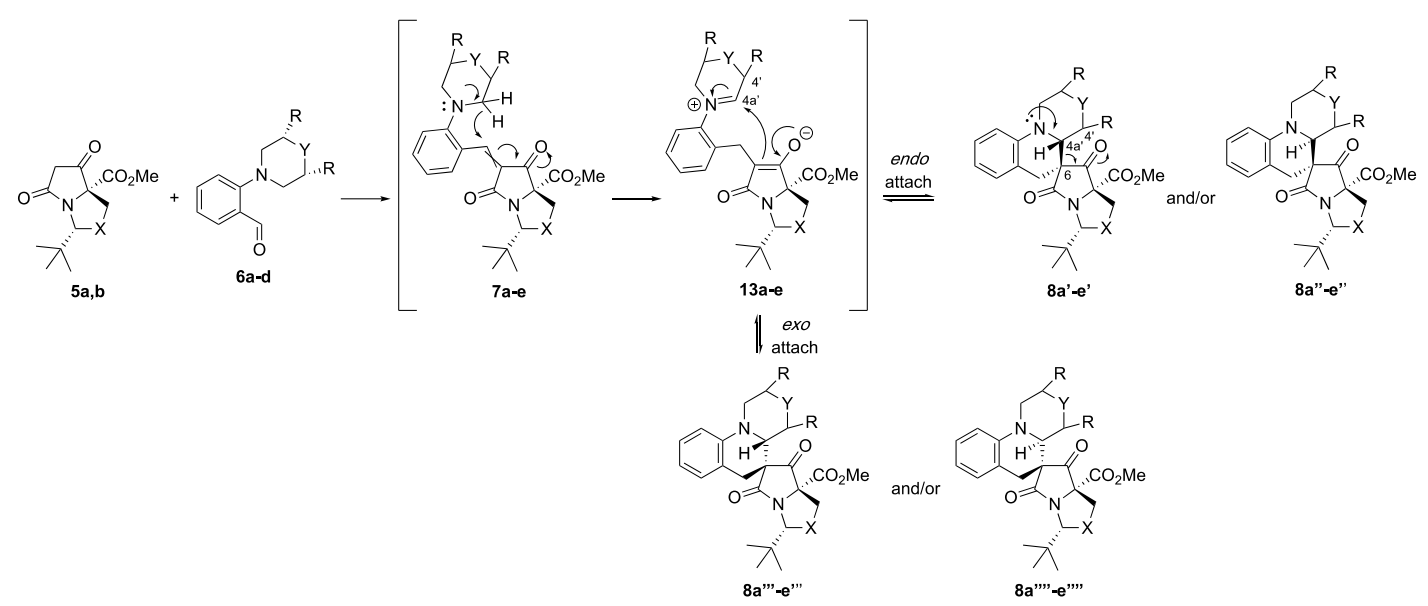

Scheme 4. Suggested mechanism of cyclisation and isomerisation.

The observed tendency of these analogues to isomerise in solution led to the question of whether the initial isolated isomer obtained via precipitation was the kinetic product of the reaction, or whether it was the only isomer to precipitate under the reaction conditions. When solid 8d was stirred in EtOAc at room temperature for $8 \mathrm{~h}$, followed by concentration under vacuum, a mixture of 4 isomers was observed by NMR spectroscopy in $\mathrm{C}_{6} \mathrm{D}_{6}$ (Figure 3 ). Redissolution of the mixture in methanol led to the precipitation of only one of the isomers, and re-analysis of the filtrates indicated the presence of 3 isomers, including the precipitated one. These results seem to indicate that while all isomers could be formed during the reaction, only one of them precipitates out of methanol, and this could partly explain the low isolation yields.

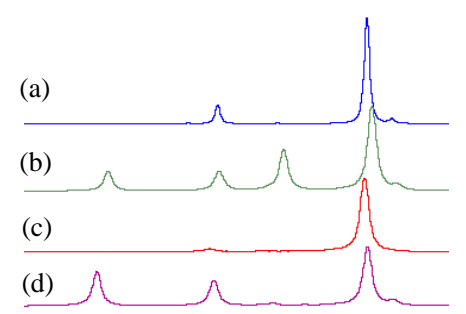

Figure 3. ${ }^{1} \mathrm{H}$ NMR spectra (in $\mathrm{C}_{6} \mathrm{D}_{6}$ ) of $H(3)$ singlet of (a) precipitate 8d, (b) 8d stirred in EtOAc for 8 h, (c) mixture concentrated and precipitated in methanol, (d) the remaining filtrates.

An attempt was made to block the reverse process by reducing the tetramate ketone group, a reaction that can be conducted easily on simpler substrates. ${ }^{40,44}$ However, reaction of $\mathbf{8 d}$ with sodium borohydride gave not the desired alcohol, but instead ring opened product 14 (Scheme 5). The enol form was clearly indicated by the $C(7){ }^{13} \mathrm{C}$ NMR chemical shift, as well as the lack of $C(6)$ proton on the ${ }^{1} \mathrm{H}$ NMR spectrum. This product probably resulted from reduction of the iminium ion formed upon ring opening, and further supports that opening of the spirocycle is possible during isomerisation (Scheme 4).

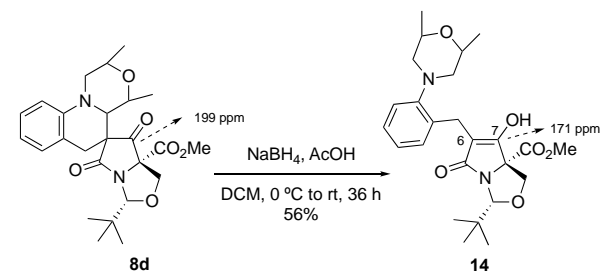

Scheme 5. Reduction of ketone 8d.

Of interest was the effect of the introduction of electron withdrawing or donating groups on the aromatic ring (Scheme 6). The nitro derivatives $\mathbf{8 f}$ and $\mathbf{8 g}$ were successfully synthesised using the previous method, but were isolated in low yields as 9:1 and 8:1 mixtures of diastereomers respectively. Nevertheless, these analogues did not isomerise in $\mathrm{C}_{6} \mathrm{D}_{6}$ over several weeks, and this is the first example of such a spirocyclic tetramate that is stable in solution. The electron withdrawing nitro groups could be deactivating the retro-Mannich reaction required to ring-open the spirocycle. A similar effect could also explain the lower yields of the transformation compared to the analogous $\mathbf{8 d}$ and $\mathbf{8 e}$, as the low electron density on the amine can also decrease the rate of the initial [1,5]-hydride shift. Enhanced stability of the nitro analogues has also been previously observed in barbiturates. ${ }^{45}$

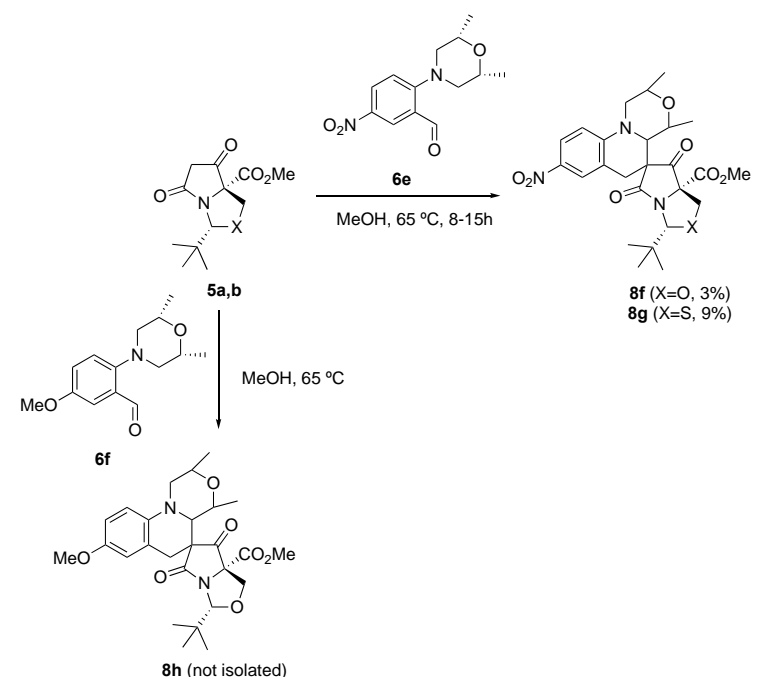

Scheme 6. Introduction of electron withdrawing $(\mathbf{8 f}, \mathbf{g})$ and donating $(\mathbf{8 h})$ groups to spirocyclic tetramates.

On the other hand, no product precipitated from the reaction mixture for the methoxy derivative $\mathbf{8 h}$. NMR analysis of the 
concentrated crude material showed a mixture of the alkylidene intermediate as well as a mixture of suspected isomers of the product, and it could be that the increased polarity of this analogue increases its solubility in methanol, preventing precipitation.

\subsection{Stereochemical identification of the spirocyclic tetramates}

Having shown that formation of spirocyclic tetramates is possible, of interest was the determination of their stereochemical identity. NOE analysis (Figure S1, SI) of the major isomer in the analogues where $\mathrm{Z}=\mathrm{H}(\mathbf{8 a - c})$ suggested that the azacycle is placed at the concave face of the bicyclic tetramate, as indicated by correlation between $H(1)-H\left(4^{\prime}\right)$ or $H(3)-H\left(4^{\prime}\right)$. Conversely, for dimethylmorpholine compounds 8d-g, correlations were observed between $H(1)-H\left(7^{\prime}\right)$ and/or $H(3)-H\left(7^{\prime}\right)$, indicative of the aromatic ring residing at the concave face of the bicycle.
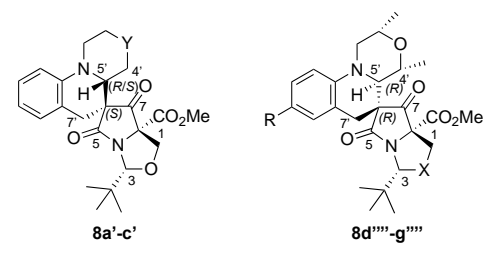

Figure 4. Major isomers of $\mathbf{8 a - g}$ predicted from NOE interactions and CLIP-HSQMBC coupling constants.
Following the stereochemical determination at the spiro-centre, we turned our attention to the configuration of the other chiral centre, 5'. For this purpose, the isolated isomers were analysed by CLIPHSQMBC. This NMR technique provides accurate measurements of long-range proton-carbon coupling constants, ${ }^{46}$ which are dependent on the dihedral angle between the atoms. ${ }^{47}$ A large ${ }^{3} \mathrm{~J}(6$ $-9 \mathrm{~Hz}$ ) represents a dihedral angle tending to $180^{\circ}$, showing a pseudo-trans relationship, while a small ${ }^{3} \mathrm{~J}$ is the result of a pseudocis relationship, with dihedral angles $20-70^{\circ}$. Samples were prepared in $\mathrm{C}_{6} \mathrm{D}_{6}$ directly before analysis. Analysis of the $H\left(5^{\prime}\right)$ $C(5)$ and the $H\left(5^{\prime}\right)-C(7)$ angles revealed that, in all compounds, $H\left(5^{\prime}\right)$ is pseudo-cis to the amide carbonyl $C(5)$, and pseudo-trans to ketone $C(7)$ (Scheme 4).

To determine the stereochemistry of the additional chiral centres (2' and 4') in the analogues with a dimethyl-morpholine, the coupling constant between the 4' and 5' protons was examined. For all analogues, the ${ }^{3} \mathrm{~J}$ was large $(8.7-9.5 \mathrm{~Hz})$, indicating a trans- configuration between the two protons, placing the methyl groups in the equatorial positions of the ring. Assuming that no epimerisation of these positions occurs during the reaction or upon isomerisation, both methyl groups should be cis- as in the starting amine 12d. (a)

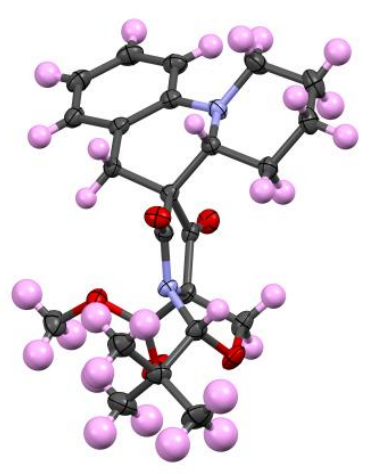

(b)

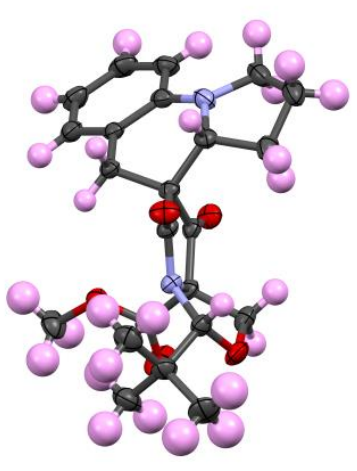

(c)

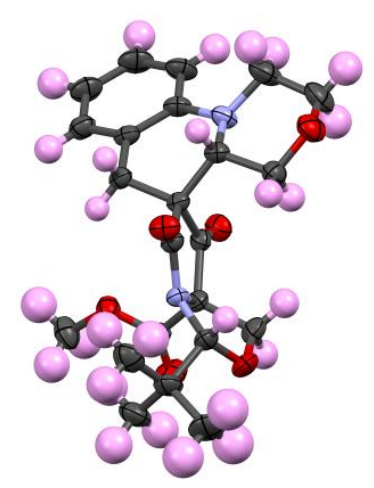

(d)

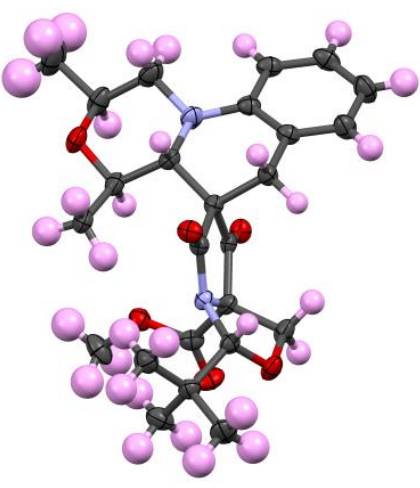

(e)

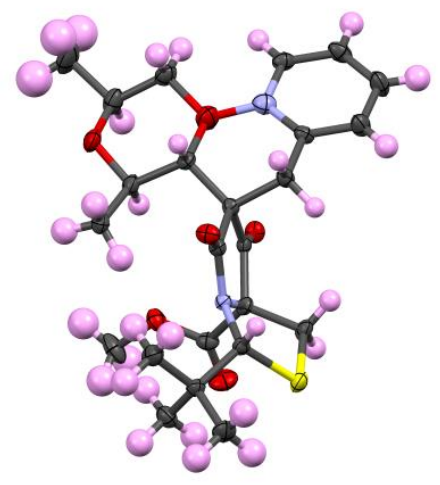

Figure 5. Structure of spirotetramates $8 \mathbf{a}(\mathrm{a}), \mathbf{8 b}(\mathrm{b}), \mathbf{8 c}(\mathrm{c}), \mathbf{8 d}(\mathrm{d})$ and $\mathbf{8 e}(\mathrm{e})$ drawn from results of single crystal X-ray diffraction studies. ${ }^{48}$ Displacement ellipsoids are drawn at $50 \%$ probability.

Therefore, the data from NOE correlations and the CLIPHSQMBC coupling constants suggest that, when $\mathrm{R}=\mathrm{H}$, the major diasteroisomer corresponds to 8a-c, while when $\mathrm{R}=\mathrm{Me}$ the structure is 8d-g (see Figure 4). The difference between these analogues could be due to the large steric hindrance when the additional methyl groups reside in the concave site of the bicycle, favouring in these latter cases the formation of a different isomer. It is worth noting that $\mathbf{8 d}$ and $\mathbf{8 e}$ had been shown to quickly 
isomerise in solution to an almost 1:1 mixture of another isomer. While the newly formed compounds could be the epimer at spirocentre $C(6)$, their complex NMR spectra impeded their precise stereochemical identification.

The absolute structure of the precipitates was determined using single crystal X-ray diffraction. ${ }^{48}$ The stereochemistry at 5', 6, 2' and 4' were indeed as predicted for all the analogues, as shown in Figure 5. The torsion angles between $H\left(5^{\prime}\right)-C(5)$ and $H\left(5^{\prime}\right)-C(7)$ correlate with those calculated with CLIP-HSQMBC (Table S1).

\subsection{Further functionalisation of the spirocyclic tetramates}

Having prepared these examples of spirocyclic tetramates, and determined their stereochemistry, of interest was whether the methodology could be extended to (1) the preparation of different tetramate bicycles; (2) the introduction of acyclic amines; and (3) further modification of the aromatic ring.

\subsubsection{Modification of the tetramate}

Using $L$-threonine, the bicyclic tetramate can be prepared with an additional methyl group at $C(1)^{49}$ (Scheme 7). Treatment of tetramate $5 \mathbf{c}$ with pyrrolidine aldehyde $\mathbf{6 b}$ resulted in no precipitation of product, and NMR analysis of the crude mixture after removal of solvent showed recovered starting material. It appears that the extra methyl at the concave face of the bicycle imposes sufficient extra steric strain that formation of the spirocycle is prevented.

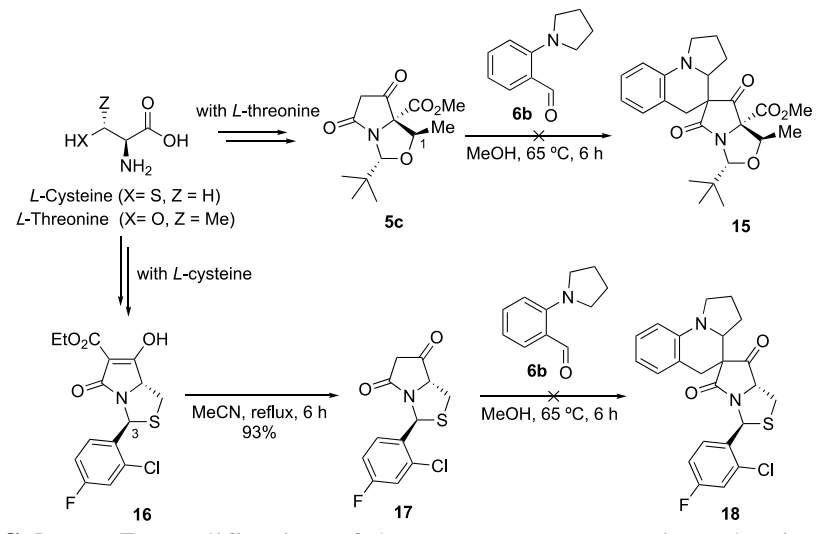

Scheme 7. Modification of the tetramate core via introduction of different groups at $C(1)$ and $C(3)$.

It has previously been shown that the tetramate $\mathbf{1 6}$ derived from cysteine may be prepared with aromatic groups at the $C(3)$ position, and that in this case tetramate cyclisation leads to isomer 16. ${ }^{50}$ To obtain the $C(6)$-unsubstituted analogue suitable for spirocyclisation, ethyl ester $\mathbf{1 6}$ was refluxed to yield decarboxylated 17. However, treatment with aldehyde $\mathbf{6 b}$ resulted again in recovered starting material. These results differ from the observed rapid reaction of $\mathbf{5 a}, \mathbf{b}$ with aldehydes $\mathbf{6 a - d}$, and illustrate the strict steric requirements of these systems, where additional hindrance can lead to a complete loss of reactivity.

\subsubsection{Introduction of acyclic amines}

Thus far, the synthesised spirotetramates were derived from cyclic amines, and of interest was the extension to the introduction of non-cyclic amines. For this purpose, $o$-aminobenzaldehydes with a range of amines 19a-j were synthesised (Scheme 8 and Table 3 ). Under the same conditions used to prepare 6a-d, yields for $\mathbf{2 1 a - j}$ were significantly reduced, and in particular as the size of the $\mathrm{R}$ groups increased. Thus, moving from $\mathrm{R}_{2}=\mathrm{Me}(\mathbf{2 1 b})$ to Et (21c) led to a large drop in reactivity even at longer reaction times, and with ${ }^{i} \operatorname{Pr}$ (21d), only starting material was recovered. Other than steric constraints, substitution with these acyclic amines could also be less entropically favourable as compared to the cyclic ones.

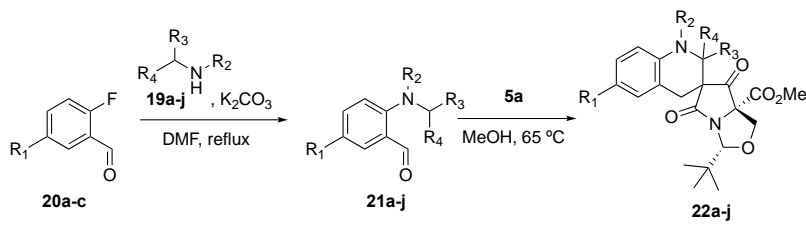

Scheme 8. Preparation of spirotetramates with acyclic amines.

Table 3. Synthesis of spirotetramates $\mathbf{2 2}$ from acyclic amines 21.

\begin{tabular}{|c|c|c|c|c|c|c|c|c|}
\hline \multirow{2}{*}{ Entry } & \multirow{2}{*}{$\mathbf{R}_{\mathbf{1}}$} & \multirow{2}{*}{$\mathbf{R}_{\mathbf{2}}$} & \multirow{2}{*}{$\mathbf{R}_{\mathbf{3}}$} & $\mathbf{R}_{\mathbf{4}}$ & $\begin{array}{c}|c| \\
\text { Yield } \\
\text { [\%] }\end{array}$ & $\begin{array}{c}\text { Time } \\
{[\mathbf{h}]}\end{array}$ & $\begin{array}{c}\text { Yield } \\
{[\%]}\end{array}$ & $\begin{array}{c}\# \\
\text { isomers }\end{array}$ \\
\hline $\mathbf{a}$ & $\mathrm{H}$ & $\mathrm{Et}$ & $\mathrm{Me}$ & $\mathrm{H}$ & 18 & 24 & 7 & $2(3: 1)$ \\
\hline $\mathbf{b}$ & $\mathrm{H}$ & $\mathrm{Me}$ & $\mathrm{Me}$ & $\mathrm{Me}$ & 16 & 48 & 0 & - \\
\hline $\mathbf{c}$ & $\mathrm{H}$ & $\mathrm{Et}$ & $\mathrm{Me}$ & $\mathrm{Me}$ & traces & 72 & - & - \\
\hline $\mathbf{d}$ & $\mathrm{H}$ & ${ }^{\mathrm{i}} \mathrm{Pr}$ & $\mathrm{Me}$ & $\mathrm{Me}$ & 0 & - & - & - \\
\hline $\mathbf{e}$ & $\mathrm{H}$ & $\mathrm{Me}$ & $\mathrm{Ph}$ & $\mathrm{H}$ & 12 & 48 & 0 & - \\
\hline $\mathbf{f}$ & $\mathrm{H}$ & $\mathrm{Et}$ & $\mathrm{Ph}$ & $\mathrm{H}$ & 25 & 48 & 0 & - \\
\hline $\mathbf{g}$ & $\mathrm{H}$ & $\mathrm{Me}$ & $\mathrm{Ph}$ & $\mathrm{Ph}$ & 0 & - & - & - \\
\hline $\mathbf{h}$ & $\mathrm{H}$ & $\mathrm{Bn}$ & $\mathrm{Ph}$ & $\mathrm{H}$ & 7 & 24 & 43 & 4 \\
\hline $\mathbf{i}$ & $\mathrm{NO}_{2}$ & $\mathrm{Et}$ & $\mathrm{Me}$ & $\mathrm{H}$ & 50 & 24 & 0 & - \\
\hline $\mathbf{j}$ & $\mathrm{Br}$ & $\mathrm{Et}$ & $\mathrm{Me}$ & $\mathrm{H}$ & 47 & 24 & 0 & - \\
\hline
\end{tabular}

With the aminobenzaldehydes in hand, spirocyclisation with $\mathbf{5 a}$ was attempted under the previous reaction conditions. Unfortunately, in this case, no solid was formed upon cooling of the reaction mixture in any of the examples, and complex reaction mixtures were isolated after solvent evaporation. While the aldehyde had been consumed in all cases, some product by NMR and MS analysis was only seen in $\mathbf{2 1 a}$ and $\mathbf{2 1} \mathbf{h}$, and these were purified by flash column chromatography to give $\mathbf{2 2 a}$ and $\mathbf{2 2 h}$ as a mixture of 2 and 4 isomers respectively. Product 22a was chosen for stereochemical determination due to its simpler NMR spectrum. NOE analysis showed no correlations between protons $H(1)$ or $H(3)$ and $H\left(5^{\prime}\right)$ or $H\left(7^{\prime}\right)$. It could be that the higher flexibility of the non-cyclic substituents leads to a less defined conformation, decreasing NOE correlations as compared to the cyclic 8a-g. Having previously proven the reliability of CLIPHSQMBC to predict the isomeric nature of 8a-g, the same technique was employed for $\mathbf{2 2 a}$. The measured ${ }^{3} \mathbf{J}$ coupling constants of the major and minor isomers were compared to the predicted torsion angles of the favoured conformations obtained from the MM2 energy minimisation tool of ChemBio3D (Table $\mathrm{S} 2$, Figure S2). In this case, the major isomer was found to place the aromatic ring in the inner face of the ring (22a), and consistent with the previous observations, the minor isomer arises from isomerisation around the spirocentre to give 22a (Figure 6).
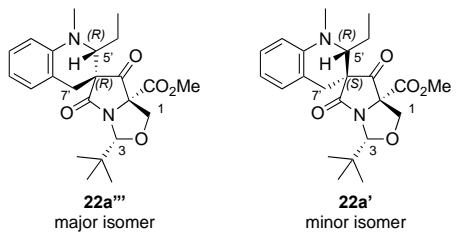

Figure 6. Isomers of 22a based on the CLIP HSQMBC results. 


\subsubsection{Modification of the aromatic ring}

Finally, it was also of interest to study the potential for functionalisation of the aromatic group. For this purpose, pyrrolidine aldehyde $\mathbf{2 3}$ was prepared, and reacted with tetramate 5a to give spiro $8 \mathbf{i}$ as a mixture of 4 diastereomers (Scheme 9). This could be converted by Suzuki coupling to phenyl derivative $\mathbf{8 j}$ as a mixture of 4 diastereomers. When the order of the reactions was reversed by performing the Suzuki reaction first on $\mathbf{2 3}$ before spirocyclisation, the same mixture of 4 diastereomers was isolated, further confirming that these spirocyclic systems isomerise in solution.

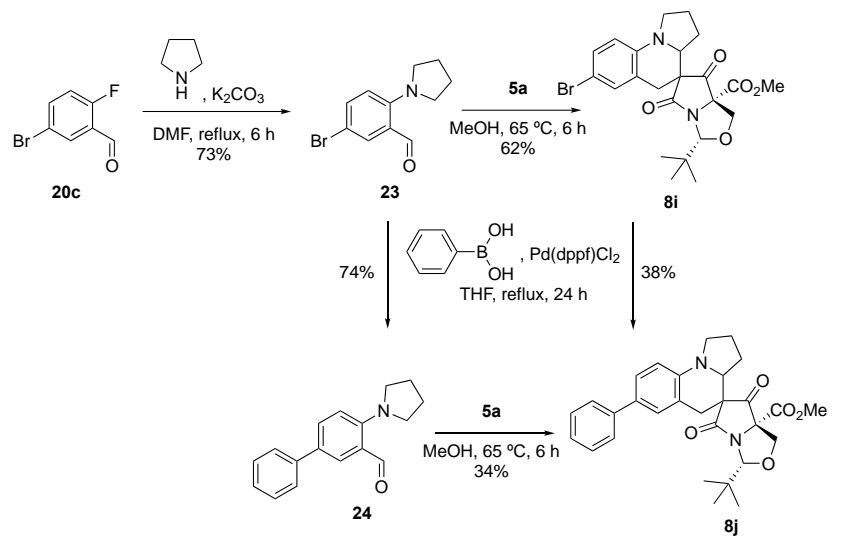

Scheme 9. Functionalisation of the aromatic ring via Suzuki coupling.

\section{Conclusion}

In summary, we have shown that the preparation of spirocyclic tetramates is possible, using a route that involves initial Knoevenagel condensation of tetramates 5a-c with aminobenzaldehydes, followed by [1,5]-hydride shift. The products, which can be isolated as one major diastereomer, were found to isomerise in solution, and the stability was dependent on the solvent and on the nature of the azacycle. This equilibration can be blocked by introducing electron withdrawing groups onto the aromatic ring. The stereochemistry of the major isolated isomers was determined using NOE and CLIP-HSQMBC correlations, and confirmed by X-ray crystallography. Further modification of the tetramate moiety of these systems was challenging, but it was possible to introduce some acyclic amines and use Suzuki coupling.

\section{Experimental section.}

General methods. All reagents were obtained from commercial sources and used without further purification. Anhydrous solvents were dried by pre-storing them over activated $3 \AA$ molecular sieves before being passed through an activated alumina column on a solvent tower under $\mathrm{N}_{2}$ pressure. Analytical thin-layer chromatography (TLC) was carried out on Merck aluminum foil backed sheets precoated with $0.2 \mathrm{~mm}$ Kielselgel 60 F254. The spots were visualized by UV irradiation $(\lambda 254 \mathrm{~nm})$ and by staining with a $\mathrm{KMnO}_{4}$ solution followed by heating. Flash column chromatography was performed on Kielselgel 60 silica gel (230-400 mesh particle size). Optical rotations were recorded at $25{ }^{\circ} \mathrm{C}$ on a polarimeter using the $\mathrm{D}$ line of sodium $(589 \mathrm{~nm})$ and a path length of $1 \mathrm{dm}$. Concentrations (c) are given in $\mathrm{g} / 100 \mathrm{~mL}$, and

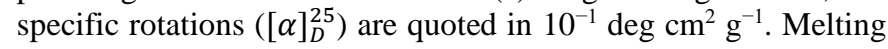
points were measured with a capillary melting point apparatus and are uncorrected. Infrared spectra were recorded on an FT-IR spectrometer; absorption maxima $\left(v_{\max }\right)$ are reported in wavenumbers $\left(\mathrm{cm}^{-1}\right)$ and only selected peaks are reported. ${ }^{1} \mathrm{H}$ NMR spectra were recorded 400, 500 and $600 \mathrm{MHz}$, and ${ }^{13} \mathrm{C} \mathrm{NMR}$ spectra at 100 and $125 \mathrm{MHz}$. Chemical shifts $\left(\delta_{\mathrm{H}}\right.$ and $\left.\delta_{\mathrm{C}}\right)$ are reported in parts per million $(\mathrm{ppm})$ and are referenced to the residual solvent peak $\left(\mathrm{CDCl}_{3}: \delta 7.26\right.$ for ${ }^{1} \mathrm{H}$ NMR and $\delta 77.2$ for ${ }^{13} \mathrm{C}$ NMR; $\mathrm{C}_{6} \mathrm{D}_{6}: \delta 7.16$ for ${ }^{1} \mathrm{H}$ NMR and $\delta 128.1$ for ${ }^{13} \mathrm{C}$ NMR). Coupling constants $(J)$ are quoted in hertz. Two-dimensional COSY, NOE and HMBC experiments were recorded at $500 \mathrm{MHz}$. Low-resolution mass spectra $(\mathrm{m} / \mathrm{z})$ were recorded using electrospray ionization (ESI); selected peaks are reported in daltons and their intensities given as percentages of the base peak. High-resolution mass spectra (HRMS) were recorded using TOF (ESI, EI or CI). In the cases where the products exist as mixtures of tautomers or diastereomers, the ratio was calculated from the ${ }^{1} \mathrm{H}$ NMR spectrum. For the NMR assignment of the spirocyclic compounds, the following numbering has been employed to facilitate comparison between analogues. The bicyclic tetramate is numbered following IUPAC guidelines.

\subsection{Synthetic procedures}

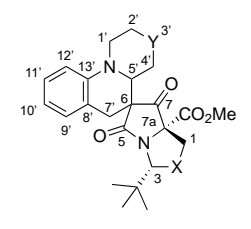

Compounds $\mathbf{5 a},{ }^{51} \mathbf{5 b},,^{52} \mathbf{5}^{49}$ and $\mathbf{1 6}{ }^{50}$ were prepared using the reported methods.

\section{General procedure A: Formation of $o$-aminobenzaldehydes}

Potassium carbonate (1.2 equiv) and the desired amine (1.2 equiv) were added to a solution of the required $o$-fluorobenzaldehyde ( 1 equiv) in DMF ( $c 0.5 \mathrm{M}$ ). The reaction mixture was heated to reflux for 3-6 hours, and then left to cool to room temperature before being diluted with water. The aqueous layer was extracted with chloroform, and the organic layer was washed with water, dried over anhydrous $\mathrm{MgSO}_{4}$ and evaporated in vacuo. The resulting oil was then eluted with $10 \%$ EtOAc in petrol through a silica plug to give the desired $o$-aminobenzaldehyde.

\section{General procedure B: Spirocyclisation}

The bicyclic tetramate 5a-c (1 equiv) was added to a solution of $o$ aminobenzaldehyde (1.2 equi) in methanol $(c 0.2 \mathrm{M})$ and the solution was heated to reflux for 2-6 h. The solution was then cooled to $0{ }^{\circ} \mathrm{C}$ and the product was precipitated out of solution. In the cases were no solid precipitates, the solvent was concentrated under reduced pressure.

\section{2-(Piperidin-1-yl)benzaldehyde (6a)}

General procedure A $(5 \mathrm{~h})$ from 2 -fluorobenzaldehyde $(170 \mu \mathrm{L}$, $1.61 \mathrm{mmol})$, piperidine $(185 \mu \mathrm{L}, 1.88 \mathrm{mmol})$ and potassium carbonate (263 mg, $1.88 \mathrm{mmol})$ to give $\mathbf{6 a}(216 \mathrm{mg}, 1.14 \mathrm{mmol}$, $71 \%)$ as a yellow oil. $\mathrm{R}_{\mathrm{f}}\left(10 \%\right.$ EtOAc in petrol) $0.53 ; v_{\max } / \mathrm{cm}^{-1}$ 1685, 1595, 1452; ${ }^{1} \mathrm{H}$ NMR (400 MHz, $\left.\mathrm{CDCl}_{3}\right) 10.30(1 \mathrm{H}, \mathrm{s}$, CHO), 7.79 (1H, dd, J 7.6, 1.8, C(6)H), $7.49(1 \mathrm{H}, \mathrm{ddd}, J$ 8.6, 7.2, 1.7, C(4)H), $7.09(1 \mathrm{H}, \mathrm{d}, J 7.8, \mathrm{C}(3) H), 7.06(1 \mathrm{H}, \mathrm{t}, J 7.4, \mathrm{C}(5) H)$, 3.05 (4H, t $J$ 5.0, C(2') $\left.H_{2}\right), 1.76\left(4 \mathrm{H}\right.$, quint, $J$ 5.7, C(3') $\left.H_{2}\right), 1.54$ - 1.65 (2H, m, C $\left.\left(4^{\prime}\right) H_{2}\right) ;{ }^{13} \mathrm{C}\left\{{ }^{1} \mathrm{H}\right\}$ NMR $\left(100 \mathrm{MHz}, \mathrm{CDCl}_{3}\right) 191.8$ (CHO), $157.1(C(2)), 134.9(C(4)), 129.3(C(6)), 128.8(C(1))$, $122.1(C(5)), 119.1(C(3)), 55.7\left(C\left(2^{\prime}\right)\right), 26.3\left(C\left(3^{\prime}\right)\right), 24.2\left(C\left(4^{\prime}\right)\right)$; $\mathrm{m} / z\left(\mathrm{ESI}^{+}\right) 190.1\left(\mathrm{MH}^{+}, 100 \%\right)$; HRMS $\left(\mathrm{EI}^{+}\right) \mathrm{m} / \mathrm{z}:[\mathrm{M}]+$ Calcd for $\mathrm{C}_{12} \mathrm{H}_{15} \mathrm{NO}$ 189.1154; Found 189.1155. Experimental data is in agreement with reported values. ${ }^{53}$

\section{2-(Pyrrolidin-1-yl)benzaldehyde (6b)}


General procedure A $(5 \mathrm{~h})$ from 2-fluorobenzaldehyde $(270 \mu \mathrm{L}$, $2.60 \mathrm{mmol})$, pyrrolidine $(260 \mu \mathrm{L}, 3.13 \mathrm{mmol})$ and potassium carbonate $(432 \mathrm{mg}, 3.13 \mathrm{mmol})$ to give $\mathbf{6 b}(441 \mathrm{mg}, 2.52 \mathrm{mmol}$, $97 \%)$ as a yellow oil. $R_{f}\left(5 \%\right.$ EtOAc in petrol) $0.69 ; v_{\max } / \mathrm{cm}^{-1} 1677$, 1560, 1491, 1478; ${ }^{1} \mathrm{H}$ NMR (400 MHz, $\left.\mathrm{CDCl}_{3}\right) 10.09(1 \mathrm{H}, \mathrm{s}$, CHO), $7.70(1 \mathrm{H}, \mathrm{dd}, J 7.8,1.8, \mathrm{C}(6) H), 7.38(1 \mathrm{H}$, ddd, $J$ 8.7, 7.0, $1.8, \mathrm{C}(4) H), 6.87-6.76(2 \mathrm{H}, \mathrm{m}, \mathrm{C}(3) H+\mathrm{C}(5) H), 3.40-3.32(4 \mathrm{H}$, tm, $\left.\mathrm{C}\left(2^{\prime}\right) H_{2}\right), 2.03-1.95\left(4 \mathrm{H}, \mathrm{m}, \mathrm{C}\left(3^{\prime}\right) H_{2}\right) ;{ }^{13} \mathrm{C}\left\{{ }^{1} \mathrm{H}\right\}$ NMR $(100$ $\left.\mathrm{MHz}, \mathrm{CDCl}_{3}\right) 190.2$ (CHO), 150.1 (C(2)), 134.3 (C(4)), 133.2 $(C(6)), 123.1(C(1)), 116.6(C(5)), 114.6(C(3)), 52.8\left(C\left(2^{\prime}\right)\right), 26.1$ $\left(C\left(3^{\prime}\right)\right) ; \mathrm{m} / \mathrm{z}\left(\mathrm{ESI}^{+}\right) 176.1\left(\mathrm{MH}^{+}, 100 \%\right) ; \operatorname{HRMS}\left(\mathrm{ESI}^{+}\right) \mathrm{m} / \mathrm{z}$ : $[\mathrm{MH}]+$ Calcd for $\mathrm{C}_{11} \mathrm{H}_{14} \mathrm{NO}$ 176.1070; Found 176.1069. Experimental data is in agreement with reported values. ${ }^{53}$

\section{2-Morpholinobenzaldehyde (6c)}

General procedure A $(5 \mathrm{~h})$ from 2-fluorobenzaldehyde $(225 \mu \mathrm{L}$, $2.02 \mathrm{mmol})$, morpholine $(210 \mu \mathrm{L}, 2.42 \mathrm{mmol})$ and potassium carbonate $(334 \mathrm{mg}, 2.42 \mathrm{mmol})$ to give $\mathbf{6 c}(268 \mathrm{mg}, 1.40 \mathrm{mmol}$, $70 \%)$ as a yellow oil. $\mathrm{R}_{\mathrm{f}}\left(20 \%\right.$ EtOAc in petrol) $0.42 ; v_{\max } / \mathrm{cm}^{-1}$ 1683, 1596; ${ }^{1} \mathrm{H}$ NMR (400 MHz, $\mathrm{CDCl}_{3}$ ) $10.33(1 \mathrm{H}, \mathrm{s}, \mathrm{CHO}), 7.81$ $(1 \mathrm{H}, \mathrm{dd}, J 7.7,1.7, \mathrm{C}(6) H), 7.54(1 \mathrm{H}, \mathrm{ddd}, J 8.1,7.3,1.7, \mathrm{C}(4) H)$, $7.17-7.12(1 \mathrm{H}, \mathrm{m}, \mathrm{C}(5) H), 7.11(1 \mathrm{H}, \mathrm{d}, J 8.2, \mathrm{C}(3) H), 3.99-3.86$ $\left(4 \mathrm{H}, \mathrm{m}, \mathrm{C}\left(3^{\prime}\right) H\right), 3.16-3.00\left(4 \mathrm{H}, \mathrm{m}, \mathrm{C}\left(2^{\prime}\right) H_{2}\right) ;{ }^{13} \mathrm{C}\left\{{ }^{1} \mathrm{H}\right\}$ NMR (100 MHz, $\left.\mathrm{CDCl}_{3}\right) 191.3$ (CHO), $155.4(C(2)), 135.2$ $(C(4)), 130.4(C(6)), 128.8(C(1)), 123.0(C(5)), 119.0(C(3)), 67.0$ $\left(C\left(3^{\prime}\right)\right)$, $54.3\left(C\left(2^{\prime}\right)\right)$; HRMS $\left(\mathrm{EI}^{+}\right) \mathrm{m} / \mathrm{z}:[\mathrm{MH}]+$ Calcd for $\mathrm{C}_{11} \mathrm{H}_{13} \mathrm{NO}_{2}$ 191.0946; Found 191.0944. Experimental data is in agreement with reported values. ${ }^{53}$

\section{2-(cis-2,6-Dimethylmorpholino)benzaldehyde (6d)}

General procedure A $(5 \mathrm{~h})$ from 2-fluorobenzaldehyde $(170 \mu \mathrm{L}$, $1.61 \mathrm{mmol}), 2$-(cis-2,6-dimethyl)morpholine $(230 \mu \mathrm{L}, 1.88 \mathrm{mmol})$ and potassium carbonate $(263 \mathrm{mg}, 1.88 \mathrm{mmol})$ to give $\mathbf{6 d}(291 \mathrm{mg}$, $1.33 \mathrm{mmol}, 82 \%)$ as an orange solid. m.p. $106-108{ }^{\circ} \mathrm{C} ; \mathrm{R}_{\mathrm{f}}(10 \%$ EtOAc in petrol) $0.45 ; v_{\max } / \mathrm{cm}^{-1} 1727,1597,1453 ;{ }^{1} \mathrm{H}$ NMR (400 $\left.\mathrm{MHz}, \mathrm{CDCl}_{3}\right) 10.31(1 \mathrm{H}, \mathrm{d}, J 0.7 \mathrm{CHO}), 7.80(1 \mathrm{H}, \mathrm{dd}, J$ 7.7, 1.7, $\mathrm{C}(4) H), 7.52(1 \mathrm{H}$, ddd, $J 8.2,7.3,1.8, \mathrm{C}(6) H), 7.12(1 \mathrm{H}, \mathrm{tt}, J 7.4$, $0.8, \mathrm{C}(5) H), 7.08(1 \mathrm{H}, \mathrm{dd}, J 8.2,0.6, \mathrm{C}(3) H), 3.91(2 \mathrm{H}, \mathrm{dqd}, J 10.0$, $\left.6.3,2.1, \mathrm{C}\left(3^{\prime}\right) H\right), 3.10-3.03\left(2 \mathrm{H}, \mathrm{m}, \mathrm{C}\left(2^{\prime}\right) H_{\mathrm{A}} \mathrm{H}_{\mathrm{B}}\right), 2.64(2 \mathrm{H}, \mathrm{dd}$, $\left.J 11.9,10.1, \mathrm{C}\left(2^{\prime}\right) \mathrm{H}_{\mathrm{A}} H_{\mathrm{B}}\right), 1.22\left(6 \mathrm{H}, \mathrm{d}, J 6.3, \mathrm{C}\left(3^{\prime}\right) \mathrm{C} H_{3}\right) ;{ }^{13} \mathrm{C}\left\{{ }^{1} \mathrm{H}\right\}$ NMR (100 MHz, $\left.\mathrm{CDCl}_{3}\right) 191.4(C \mathrm{HO}), 155.2$ (C(2)), 135.2 (C(6)), $130.3(C(4)), 128.8(C(1)), 122.9(C(5)), 119.1(C(3)), 71.9$ $\left(C\left(3^{\prime}\right)\right), 59.8\left(C\left(2^{\prime}\right)\right), 19.0\left(\mathrm{C}\left(3^{\prime}\right) C \mathrm{H}_{3}\right) ; \mathrm{m} / \mathrm{z}\left(\mathrm{ESI}^{+}\right) 220.1\left(\mathrm{MH}^{+}\right.$, $100 \%$ ); HRMS (ESI-TOF) m/z: [MH]+ Calcd for $\mathrm{C}_{13} \mathrm{H}_{18} \mathrm{NO}_{2}$ 220.1332; Found 220.1329. Experimental data is in agreement with reported values. ${ }^{54}$

\section{2-(cis-2,6-Dimethylmorpholino)-5-nitrobenzaldehyde (6e)}

General procedure A $(6 \mathrm{~h})$ from 2-fluoro-5-nitrobenzaldehyde (1.50 g, $8.87 \mathrm{mmol}), 2$-(cis-2,6-dimethyl)morpholine (1.32 mL, $10.6 \mathrm{mmol})$ and potassium carbonate $(1.47 \mathrm{~g}, 10.6 \mathrm{mmol})$ to give 6e $(1.91 \mathrm{~g}, 7.23 \mathrm{mmol}, 82 \%)$ as a yellow solid. m.p. $120-121^{\circ} \mathrm{C}$; $\mathrm{R}_{\mathrm{f}}\left(50 \%\right.$ EtOAc in petrol) $0.68 ; v_{\max } / \mathrm{cm}^{-1} 2871,1687,1508,1335$; ${ }^{1} \mathrm{H}$ NMR $\left(400 \mathrm{MHz}, \mathrm{CDCl}_{3}\right) 10.06(1 \mathrm{H}, \mathrm{s}, \mathrm{CHO}), 8.61(1 \mathrm{H}, \mathrm{d}, J$ 2.8, C(6)H), $8.29(1 \mathrm{H}, \mathrm{dd}, J 9.1,2.8, \mathrm{C}(4) H), 7.06(1 \mathrm{H}, \mathrm{d}, J 9.1$, $\mathrm{C}(3) H), 3.91\left(2 \mathrm{H}, \mathrm{dqd}, J 10.2,6.2,2.1, \mathrm{C}\left(3^{\prime}\right) H\right), 3.40-3.22(2 \mathrm{H}$, m, $\left.\mathrm{C}\left(2^{\prime}\right) H_{\mathrm{A}} \mathrm{H}_{B}\right), 2.82\left(2 \mathrm{H}, \mathrm{dd}, J 12.4,10.2, \mathrm{C}\left(2^{\prime}\right) \mathrm{H}_{\mathrm{A}} H_{\mathrm{B}}\right), 1.24(6 \mathrm{H}$, d, $J$ 6.3, C(3') $\left.\mathrm{CH}_{3}\right) ;{ }^{13} \mathrm{C}\left\{{ }^{1} \mathrm{H}\right\}$ NMR $\left(100 \mathrm{MHz}, \mathrm{CDCl}_{3}\right) 188.4$ (CHO), $157.5(C(2)), 141.1(C(5)), 129.6(C(4)), 129.1(C(6))$, $126.0(C(1)), 118.4(C(3)), 71.7\left(C\left(3^{\prime}\right)\right), 58.6\left(C\left(2^{\prime}\right)\right), 18.8$ $\left(\left(\mathrm{C}\left(3^{\prime}\right) C \mathrm{H}_{3}\right) ; \mathrm{m} / z\left(\mathrm{ESI}^{+}\right) 265.1\left(\mathrm{MH}^{+}, 28 \%\right)\right.$; HRMS $\left(\mathrm{ESI}^{+}\right) \mathrm{m} / \mathrm{z}$ : $[\mathrm{MH}]+$ Calcd for $\mathrm{C}_{13} \mathrm{H}_{16} \mathrm{~N}_{2} \mathrm{O}_{4}$ 265.1183; Found 265.1183. Experimental data is in agreement with reported values. ${ }^{20}$

2-(cis-2,6-Dimethylmorpholino)-5-methoxybenzaldehyde (6f)
General procedure A $(8 \mathrm{~h})$ from 2-fluoro-5-methoxybenzaldehyde (1.03 mL, $8.30 \mathrm{mmol}), 2$-(cis-2,6-dimethyl)morpholine $(880 \mu \mathrm{L}$, $9.73 \mathrm{mmol})$ and potassium carbonate $(1.35 \mathrm{~g}, 9.73 \mathrm{mmol})$ to give 6f (408 mg, $1.64 \mathrm{mmol}, 20 \%)$ as a yellow oil. $\mathrm{R}_{\mathrm{f}}(50 \%$ EtOAc in petrol) $0.50 ; v_{\max } / \mathrm{cm}^{-1} 2868,1684 ;{ }^{1} \mathrm{H} \mathrm{NMR}\left(400 \mathrm{MHz}, \mathrm{CDCl}_{3}\right)$ $10.42(1 \mathrm{H}, \mathrm{s}, \mathrm{CHO}), 7.33-7.31(1 \mathrm{H}, \mathrm{m}, \mathrm{C}(6) H), 7.15-7.08(2 \mathrm{H}$, $\mathrm{m}, \mathrm{C}(3) H+\mathrm{C}(4) H), 3.89\left(2 \mathrm{H}, \mathrm{dqd}, J 12.5,6.2,1.9, \mathrm{C}\left(3^{\prime}\right) H\right), 3.82$ $\left(3 \mathrm{H}, \mathrm{s}, \mathrm{OCH}_{3}\right), 2.95\left(2 \mathrm{H}, \mathrm{d}, J 10.9, \mathrm{C}\left(2^{\prime}\right) H_{\mathrm{A}} \mathrm{H}_{\mathrm{B}}\right), 2.61(2 \mathrm{H}, \mathrm{t}, J 10.8$, $\left.\mathrm{C}\left(2^{\prime}\right) \mathrm{H}_{\mathrm{A}} H_{\mathrm{B}}\right), 1.21\left(6 \mathrm{H}, \mathrm{d}, J 6.3, \mathrm{C}\left(3^{\prime}\right) \mathrm{CH}_{3}\right) ;{ }^{13} \mathrm{C}\left\{{ }^{1} \mathrm{H}\right\} \mathrm{NMR}(100$ $\left.\mathrm{MHz}, \mathrm{CDCl}_{3}\right) 191.6(C \mathrm{HO}), 156.0(C(5)), 149.8(C(2)), 130.2$ $(C(1)), 122.8(C(4)), 121.4(C(3)), 111.3(C(6)), 72.0\left(C\left(3^{\prime}\right)\right), 60.4$ $\left(C\left(2^{\prime}\right)\right), 55.8\left(\mathrm{OCH}_{3}\right), 19.0\left(\mathrm{C}\left(3^{\prime}\right) C \mathrm{H}_{3}\right) ; \mathrm{m} / z\left(\mathrm{ESI}^{+}\right) 250.1\left(\mathrm{MH}^{+}\right.$, $88 \%$ ); HRMS $\left(\mathrm{ESI}^{+}\right) \mathrm{m} / \mathrm{z}$ : $[\mathrm{MH}]+$ Calcd for $\mathrm{C}_{14} \mathrm{H}_{20} \mathrm{NO}_{3} 250.1438$; Found 250.1438 .

Methyl (3'R,4aR,5S,7a'R)-3'-(tert-butyl)-5',7'-dioxo-2,3,4,4atetrahydro-1H,1'H,3' $H, 5^{\prime} H, 6 H$-spiro[pyrido[1,2-a]quinoline$5,6^{\prime}$-pyrrolo[1,2-c]oxazole]-7a' $\left(7^{\prime} H\right)$-carboxylate (major isomer of 8a)

General procedure B $(6 \mathrm{~h})$ from tetramate $\mathbf{5 a}(500 \mathrm{mg}, 1.96 \mathrm{mmol})$ and $o$-aminobenzaldehyde $\mathbf{6 a}(408 \mathrm{mg}, 2.15 \mathrm{mmol})$ to give spirocycle 8a (309 mg, $0.724 \mathrm{mmol}, 37 \%$ ) as a red solid. m.p. 185 ${ }^{\circ} \mathrm{C} ; \mathrm{R}_{\mathrm{f}}\left(25 \%\right.$ EtOAc in petrol) $0.66 ; v_{\max } / \mathrm{cm}^{-1} 1774,1746,1720$, 1497, 1458, 1497, 1267; ${ }^{1} \mathrm{H}$ NMR $\left(600 \mathrm{MHz}, \mathrm{C}_{6} \mathrm{D}_{6}\right) 7.09$ (1H, t, $J$ 7.6, C(11')H), $6.82\left(1 \mathrm{H}, \mathrm{d}, J 7.4, \mathrm{C}\left(9^{\prime}\right) H\right), 6.72(1 \mathrm{H}, \mathrm{d}, J 8.4$, $\left.\mathrm{C}\left(12^{\prime}\right) H\right), 6.67\left(1 \mathrm{H}, \mathrm{t}, J 7.3, \mathrm{C}\left(10^{\prime}\right) H\right), 5.11(1 \mathrm{H}, \mathrm{s}, \mathrm{C}(3) H), 4.75$ $\left(1 \mathrm{H}, \mathrm{d}, J 8.9, \mathrm{C}(1) H_{\mathrm{A}} \mathrm{H}_{\mathrm{B}}\right), 3.76-3.70\left(1 \mathrm{H}, \mathrm{m}, \mathrm{C}\left(1^{\prime}\right) H_{\mathrm{A}} \mathrm{H}_{\mathrm{B}}\right), 3.41-$ $3.36\left(2 \mathrm{H}, \mathrm{m}, \mathrm{C}\left(7^{\prime}\right) H_{\mathrm{A}} \mathrm{H}_{\mathrm{B}}+\mathrm{C}\left(5^{\prime}\right) H\right), 3.26(1 \mathrm{H}, \mathrm{d}, J 15.8$, $\left.\mathrm{C}\left(7^{\prime}\right) \mathrm{H}_{\mathrm{A}} H_{\mathrm{B}}\right), 3.20\left(1 \mathrm{H}, \mathrm{d}, J 8.8, \mathrm{C}(1) \mathrm{H}_{\mathrm{A}} H_{\mathrm{B}}\right), 3.12\left(3 \mathrm{H}, \mathrm{s}, \mathrm{CO}_{2} \mathrm{CH}_{3}\right)$, $2.42\left(1 \mathrm{H}, \mathrm{td}, J 12.3,4.4, \mathrm{C}\left(1^{\prime}\right) \mathrm{H}_{\mathrm{A}} H_{\mathrm{B}}\right), 1.50-1.44(2 \mathrm{H}, \mathrm{m}$, $\left.\mathrm{C}\left(3^{\prime}\right) \mathrm{H}_{\mathrm{A}} H_{\mathrm{B}}+\mathrm{C}\left(4^{\prime}\right) H_{\mathrm{A}} \mathrm{H}_{\mathrm{B}}\right), 1.32-1.27\left(2 \mathrm{H}, \mathrm{m}, \mathrm{C}\left(2^{\prime}\right) H_{\mathrm{A}} H_{\mathrm{B}}\right), 1.12$ $-1.06\left(1 \mathrm{H}, \mathrm{m}, \mathrm{C}\left(3^{\prime}\right) H_{\mathrm{A}} \mathrm{H}_{\mathrm{B}}\right), 1.04\left(9 \mathrm{H}, \mathrm{s}, \mathrm{C}\left(\mathrm{CH}_{3}\right)_{3}\right), 1.00-0.96$ $\left(1 \mathrm{H}, \mathrm{m}, \mathrm{C}\left(4^{\prime}\right) \mathrm{H}_{\mathrm{A}} H_{\mathrm{B}}\right) ;{ }^{13} \mathrm{C}\left\{{ }^{1} \mathrm{H}\right\}$ NMR $\left(100 \mathrm{MHz}, \mathrm{C}_{6} \mathrm{D}_{6}\right) 199.6$ $(C(7)), 178.6(C(5)), 167.6\left(C_{2} \mathrm{CH}_{3}\right), 146.2\left(C\left(13^{\prime}\right)\right), 129.4$

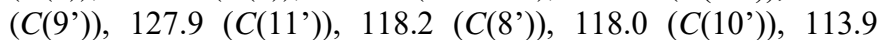
(C(12')), 99.2 (C(3)), $78.5(C(7 \mathrm{a})), 68.2(C(1)), 59.0\left(C\left(5^{\prime}\right)\right), 57.7$ $(C(6)), 52.9\left(\mathrm{CO}_{2} \mathrm{CH}_{3}\right), 48.7\left(C\left(1^{\prime}\right)\right), 36.9\left(C\left(7^{\prime}\right)\right), 35.7\left(C\left(\mathrm{CH}_{3}\right)_{3}\right)$, $29.3\left(C\left(4^{\prime}\right)\right), 25.6\left(C\left(2^{\prime}\right)\right), 25.0\left(C\left(\mathrm{CH}_{3}\right)_{3}\right), 24.5\left(C\left(3^{\prime}\right)\right) ; \mathrm{m} / z\left(\mathrm{ESI}^{+}\right)$ $427.2\left(\mathrm{MH}^{+}, 100 \%\right)$; HRMS $\left(\mathrm{ESI}^{+}\right) \mathrm{m} / \mathrm{z}:[\mathrm{MH}]^{+}$Calcd for $\mathrm{C}_{24} \mathrm{H}_{31} \mathrm{~N}_{2} \mathrm{O}_{5}$ 427.2228; Found 427.2212. Single Crystal Data: $\mathrm{C}_{24} \mathrm{H}_{30} \mathrm{~N}_{2} \mathrm{O}_{5}$, tetragonal, $\mathrm{I}_{1}, a=16.4812(2), c=16.0775(3) \AA, V=$ 4367.13(11) $\AA^{3}, \quad$ Data/restraints/parameters 4521/1/281, $\mathrm{R}_{\text {int }}=0.029$, Flack $x=0.00(11)$, Final $\mathrm{R}_{1}=0.0272, \quad \mathrm{wR}_{2}=0.0689$ (I $>2 \mathrm{~s}(\mathrm{I}))$.

\section{Methyl (3aR,3'R,4S,7a'R)-3'-(tert-butyl)-5',7'-dioxo-1,2,3,3a- tetrahydro-1' $H, 3^{\prime} H, 5 H, 5^{\prime} H$-spiro[pyrrolo[1,2-a]quinoline- 4,6'-pyrrolo[1,2-c]oxazole]-7a' (7' H)-carboxylate (major isomer of $8 \mathrm{~b}$ )}

General procedure B $(6 \mathrm{~h})$ from tetramate $\mathbf{5 a}(500 \mathrm{mg}, 1.96 \mathrm{mmol})$ and $o$-aminobenzaldehyde $\mathbf{6 b}(420 \mathrm{mg}, 2.15 \mathrm{mmol})$ to give spirocycle $\mathbf{8 b}$ (474 mg, $1.15 \mathrm{mmol}, 58 \%)$ as an orange solid. m.p. $188{ }^{\circ} \mathrm{C} ; \mathrm{R}_{\mathrm{f}}\left(25 \%\right.$ EtOAc in petrol) $0.70 ; v_{\max } / \mathrm{cm}^{-1} 1745,1717 ;{ }^{1} \mathrm{H}$ NMR (600 MHz, C $\left.{ }_{6} \mathrm{D}_{6}\right) 7.20\left(1 \mathrm{H}, \mathrm{t}, J 7.6, \mathrm{C}\left(11^{\prime}\right) H\right), 6.93(1 \mathrm{H}, \mathrm{d}$, $J$ 7.4, C(9')H), $6.69(1 \mathrm{H}, \mathrm{t}, J$ 7.4, C(10')H), $6.49(1 \mathrm{H}, \mathrm{d}, J$ 8.0, $\left.\mathrm{C}\left(12^{\prime}\right) H\right), 5.15(1 \mathrm{H}, \mathrm{s}, \mathrm{C}(3) H), 4.76\left(1 \mathrm{H}, \mathrm{d}, J 8.9, \mathrm{C}(1) H_{\mathrm{A}} \mathrm{H}_{B}\right), 3.82$ $\left(1 \mathrm{H}, \mathrm{dd}, J 10.2,5.6, \mathrm{C}\left(5^{\prime}\right) H\right), 3.47\left(1 \mathrm{H}, \mathrm{d}, J 15.9, \mathrm{C}\left(7^{\prime}\right) H_{\mathrm{A}} \mathrm{H}_{\mathrm{B}}\right), 3.23$ $\left(1 \mathrm{H}, \mathrm{d}, J 15.9, \mathrm{C}\left(7^{\prime}\right) \mathrm{H}_{\mathrm{A}} H_{\mathrm{B}}\right), 3.17\left(1 \mathrm{H}, \mathrm{d}, J 8.9, \mathrm{C}(1) \mathrm{H}_{\mathrm{A}} H_{\mathrm{B}}\right), 3.12-$ $3.09\left(1 \mathrm{H}, \mathrm{m}, \mathrm{C}\left(1^{\prime}\right) H_{\mathrm{A}} \mathrm{H}_{B}\right), 3.09\left(3 \mathrm{H}, \mathrm{s}, \mathrm{CO}_{2} \mathrm{CH}_{3}\right), 2.94(1 \mathrm{H}, \mathrm{td}, J$ 9.3, 7.0, C(1') $\left.\mathrm{H}_{\mathrm{A}} H_{\mathrm{B}}\right), 1.71\left(1 \mathrm{H}, \mathrm{dtd}, J 11.4,6.2,1.7, \mathrm{C}\left(4^{\prime}\right) H_{\mathrm{A}} \mathrm{H}_{B}\right)$, $1.48-1.37\left(2 \mathrm{H}, \mathrm{m}, \mathrm{C}\left(2^{\prime}\right) H_{A} H_{B}\right), 1.04\left(9 \mathrm{H}, \mathrm{s}, \mathrm{C}\left(\mathrm{CH}_{3}\right)_{3}\right), 0.97-0.88$ (1H, m, C $\left.\left(4^{\prime}\right) \mathrm{H}_{\mathrm{A}} H_{\mathrm{B}}\right) ;{ }^{13} \mathrm{C}\left\{{ }^{1} \mathrm{H}\right\}$ NMR (100 MHz, $\left.\mathrm{C}_{6} \mathrm{D}_{6}\right) 199.3$ $(C(7)), 178.5(C(5)), 167.1\left(C_{2} \mathrm{CH}_{3}\right), 143.9\left(C\left(13^{\prime}\right)\right), 128.6$ (C(9')), 128.2 (C(11')), $115.9\left(C\left(10^{\prime}\right)\right), 115.3\left(C\left(8^{\prime}\right)\right), 110.9$ (C(12')), $98.7(C(3)), 78.1(C(7 \mathrm{a})), 67.8(C(1)), 60.1\left(C\left(5^{\prime}\right)\right), 52.6$ $\left(\mathrm{CO}_{2} \mathrm{CH}_{3}\right), 52.4(C(6)), 47.3\left(C\left(1^{\prime}\right)\right), 37.7\left(C\left(7^{\prime}\right)\right), 35.3\left(C\left(\mathrm{CH}_{3}\right)_{3}\right)$, 
$28.6\left(C\left(4^{\prime}\right)\right), 24.6\left(\mathrm{C}\left(\mathrm{CH}_{3}\right)_{3}\right), 23.5\left(C\left(2^{\prime}\right)\right) ; \mathrm{m} / z\left(\mathrm{ESI}^{+}\right) 413.2\left(\mathrm{MH}^{+}\right.$, $100 \%)$; HRMS $\left(\mathrm{ESI}^{+}\right) \mathrm{m} / \mathrm{z}:[\mathrm{MH}]+$ Calcd for $\mathrm{C}_{23} \mathrm{H}_{29} \mathrm{~N}_{2} \mathrm{O}_{5}$ 413.2071; Found 413.2065. Single Crystal Data: $\mathrm{C}_{23} \mathrm{H}_{28} \mathrm{~N}_{2} \mathrm{O}_{5}$, tetragonal, $\quad \mathrm{I}_{4}, \quad a=16.4674(2), \quad c=15.6590(4) \AA$, $V=4246.33(13) \AA^{3}, \quad$ Data/restraints/parameters $\quad 4405 / 1 / 272$, $\mathrm{R}_{\text {int }}=0.030$, Flack $x=0.07(12)$, Final $\mathrm{R}_{1}=0.0277, \quad \mathrm{w}_{2}=0.0706$ (I>2s(I))

Methyl (3R,4a' $S, 6 S, 7 \mathrm{a} R)$-3-(tert-butyl)-5,7-dioxo-1',2',4',4a'tetrahydro-1H,3H,5H,6' $H$-spiro[pyrrolo[1,2-c] ]oxazole-6,5'$[1,4]$ oxazino $[4,3-a]$ quinoline]-7a(7H)-carboxylate (major isomer of $8 \mathrm{c}$ )

General procedure B (12 h) from tetramate 5a (500 mg, 1.96 $\mathrm{mmol})$ and $o$-aminobenzaldehyde $\mathbf{6 c}(440 \mathrm{mg}, 2.15 \mathrm{mmol})$ to give spirocycle 8c (53 mg, $0.12 \mathrm{mmol}, 7 \%)$ as a red solid. m.p. 172 $175{ }^{\circ} \mathrm{C} ; \mathrm{R}_{\mathrm{f}}\left(25 \%\right.$ EtOAc in petrol) $0.46 ; v_{\max } / \mathrm{cm}^{-1} 1773,1747$, 1718; ${ }^{1} \mathrm{H}$ NMR $\left(500 \mathrm{MHz}, \mathrm{C}_{6} \mathrm{D}_{6}\right) 7.09\left(1 \mathrm{H}, \mathrm{t}, J 7.8, \mathrm{C}\left(11^{\prime}\right) H\right), 6.80$ (1H, d, J 7.4, C(9')H), $6.70(1 \mathrm{H}, \mathrm{t}, J$ 7.0, C(10')H), $6.57(1 \mathrm{H}, \mathrm{d}, J$ $\left.8.3, \mathrm{C}\left(12^{\prime}\right) H\right), 4.95(1 \mathrm{H}, \mathrm{s}, \mathrm{C}(3) H), 4.68\left(1 \mathrm{H}, \mathrm{d}, J 9.0, \mathrm{C}(1) H_{\mathrm{A}} \mathrm{H}_{\mathrm{B}}\right)$, $3.82\left(1 \mathrm{H}, \mathrm{dd}, J 10.1,2.9, \mathrm{C}\left(4^{\prime}\right) H_{\mathrm{A}} \mathrm{H}_{\mathrm{B}}\right), 3.63(1 \mathrm{H}, \mathrm{dd}, J 10.2,3.0$, $\left.\mathrm{C}\left(5^{\prime}\right) H\right), 3.58-3.54\left(1 \mathrm{H}, \mathrm{m}, \mathrm{C}\left(2^{\prime}\right) H_{\mathrm{A}} \mathrm{H}_{\mathrm{B}}\right), 3.37(1 \mathrm{H}, \mathrm{d}, J 16.2$, $\left.\mathrm{C}\left(7^{\prime}\right) H_{\mathrm{A}} \mathrm{H}_{\mathrm{B}}\right), 3.34\left(1 \mathrm{H}, \mathrm{td}, J 11.5,3.0, \mathrm{C}\left(2^{\prime}\right) \mathrm{H}_{\mathrm{A}} H_{\mathrm{B}}\right), 3.22(1 \mathrm{H}, \mathrm{d}, J$ $\left.16.2, \mathrm{C}\left(7^{\prime}\right) \mathrm{H}_{\mathrm{A}} H_{\mathrm{B}}\right), 3.21-3.17\left(1 \mathrm{H}, \mathrm{m}, \mathrm{C}\left(1^{\prime}\right) H_{\mathrm{A}} \mathrm{H}_{\mathrm{B}}\right), 3.08(3 \mathrm{H}, \mathrm{s}$, $\left.\mathrm{CO}_{2} \mathrm{CH}_{3}\right), 3.09-3.04\left(2 \mathrm{H}, \mathrm{m}, \mathrm{C}(1) \mathrm{H}_{\mathrm{A}} H_{\mathrm{B}}+\mathrm{C}\left(4^{\prime}\right) \mathrm{H}_{\mathrm{A}} H_{\mathrm{B}}\right), 2.60(1 \mathrm{H}$, td, $\left.J 11.9,3.7, \mathrm{C}\left(1^{\prime}\right) \mathrm{H}_{\mathrm{A}} H_{\mathrm{B}}\right), 1.00\left(9 \mathrm{H}, \mathrm{s}, \mathrm{C}\left(\mathrm{CH}_{3}\right)_{3}\right) ;{ }^{13} \mathrm{C}\left\{{ }^{1} \mathrm{H}\right\} \mathrm{NMR}$ $\left(125 \mathrm{MHz}, \mathrm{C}_{6} \mathrm{D}_{6}\right) 199.6(C(7)), 177.8(C(5)), 167.5\left(C_{2} \mathrm{CH}_{3}\right)$, $145.2\left(C\left(13^{\prime}\right)\right), 129.6\left(C\left(9^{\prime}\right)\right), 128.4\left(C\left(11^{\prime}\right)\right), 118.8\left(C\left(10^{\prime}\right)\right)$, $118.2\left(C\left(8^{\prime}\right)\right), 113.1\left(C\left(12^{\prime}\right)\right), 99.1(C(3)), 78.6(C(7 \mathrm{a})), 68.4$ $(C(1)), 68.0\left(C\left(4^{\prime}\right)\right), 66.9\left(C\left(2^{\prime}\right)\right), 57.5\left(C\left(5^{\prime}\right)\right), 53.8(C(6)), 53.0$ $\left(\mathrm{CO}_{2} \mathrm{CH}_{3}\right), 46.4\left(C\left(1^{\prime}\right)\right), 36.5\left(C\left(7^{\prime}\right)\right), 35.6\left(C\left(\mathrm{CH}_{3}\right)_{3}\right), 25.0$ $\left(\mathrm{C}\left(\mathrm{CH}_{3}\right)_{3}\right) ; \mathrm{m} / \mathrm{z}\left(\mathrm{ESI}^{+}\right) 429.2\left(\mathrm{MH}^{+}, 100 \%\right) ; \mathrm{HRMS}\left(\mathrm{ESI}^{+}\right) \mathrm{m} / \mathrm{z}$ : $[\mathrm{MH}]+$ Calcd for $\mathrm{C}_{23} \mathrm{H}_{29} \mathrm{~N}_{2} \mathrm{O}_{6} 429.2020$; Found 429.2017. Single Crystal Data: $\mathrm{C}_{23} \mathrm{H}_{28} \mathrm{~N}_{2} \mathrm{O}_{6}$, tetragonal, I4 ${ }_{1}, \quad a=16.3993(2)$, $c=15.9819(3) \AA, \quad V=4298.12(13) \AA^{3}$, Data/restraints/parameters $4195 / 1 / 281, \mathrm{R}_{\mathrm{int}}=0.017$, Flack $x=0.01(13)$, Final $\mathrm{R}_{1}=0.0287$, $\mathrm{wR}_{2}=0.0806(\mathrm{I}>2 \mathrm{~s}(\mathrm{I})){ }^{55}$

Methyl (2' $\left.S, 3 R, 4^{\prime} R, 4 \mathrm{a}^{\prime} R, 6 R, 7 \mathrm{a} R\right)$-3-(tert-butyl)-2',4'-dimethyl5,7-dioxo-1',2',4',4a'-tetrahydro-1 $\mathrm{H}, 3 \mathrm{H}, 5 \mathrm{H}, \mathbf{6}^{\prime} \mathrm{H}$ spiro[pyrrolo[1,2-c] oxazole-6,5'-[1,4] oxazino[4,3a]quinoline]-7a(7H)-carboxylate (major isomer of 8d)

General procedure B $(8 \mathrm{~h})$ from tetramate $5 \mathrm{a}(2.25 \mathrm{~g}, 8.82 \mathrm{mmol})$ and $o$-aminobenzaldehyde $\mathbf{6 d}(2.13 \mathrm{~g}, 9.70 \mathrm{mmol})$ to give spirocycle 8d (1.24 g, $2.72 \mathrm{mmol}, 31 \%)$ as a pink solid. m.p. 190 ${ }^{\circ} \mathrm{C} ; \mathrm{R}_{\mathrm{f}}\left(50 \%\right.$ EtOAc in petrol) $0.88 ; v_{\max } / \mathrm{cm}^{-1} 1773,1745,1721 ;{ }^{1} \mathrm{H}$ NMR $\left(600 \mathrm{MHz}, \mathrm{C}_{6} \mathrm{D}_{6}, 8: 2\right.$ mixture of diastereomers) major isomer 8d"',' $7.15-7.11\left(1 \mathrm{H}, \mathrm{m}, \mathrm{C}\left(11^{\prime}\right) H\right), 6.80(1 \mathrm{H}, \mathrm{d}, J 7.4,1.8$, $\left.\mathrm{C}\left(9^{\prime}\right) H\right), 6.76\left(1 \mathrm{H}, \mathrm{td}, J 7.2,0.9, \mathrm{C}\left(10^{\prime}\right) H\right), 6.61(1 \mathrm{H}, \mathrm{d}, J 8.4$, $\left.\mathrm{C}\left(12^{\prime}\right) H\right), 5.05(1 \mathrm{H}, \mathrm{s}, \mathrm{C}(3) H), 4.65\left(1 \mathrm{H}, \mathrm{d}, J 8.9, \mathrm{C}(1) H_{\mathrm{A}} \mathrm{H}_{\mathrm{B}}\right), 3.72$ $\left(1 \mathrm{H}, \mathrm{dq}, J 9.5,6.0, \mathrm{C}\left(4^{\prime}\right) H\right), 3.48(1 \mathrm{H}, \mathrm{dqd}, J 12.4,6.1,2.7$, $\left.\mathrm{C}\left(2^{\prime}\right) H\right), 3.42\left(1 \mathrm{H}, \mathrm{dd}, J 12.0,2.7, \mathrm{C}\left(1^{\prime}\right) H_{\mathrm{A}} \mathrm{H}_{B}\right), 3.31(1 \mathrm{H}, \mathrm{d}, J 16.5$, $\left.\mathrm{C}\left(7^{\prime}\right) H_{\mathrm{A}} \mathrm{H}_{\mathrm{B}}\right), 3.30\left(1 \mathrm{H}, \mathrm{d}, J 9.5, \mathrm{C}\left(5^{\prime}\right) H\right), 3.18\left(3 \mathrm{H}, \mathrm{s}, \mathrm{CO}_{2} \mathrm{CH}_{3}\right)$, $3.02\left(1 \mathrm{H}, \mathrm{d}, J 8.9, \mathrm{C}(1) \mathrm{H}_{\mathrm{A}} H_{B}\right), 2.40\left(1 \mathrm{H}, \mathrm{t}, J 11.2, \mathrm{C}\left(1^{\prime}\right) \mathrm{H}_{\mathrm{A}} H_{B}\right)$, $2.38\left(1 \mathrm{H}, \mathrm{d}, J 16.8, \mathrm{C}\left(7^{\prime}\right) \mathrm{H}_{\mathrm{A}} H_{B}\right), 1.69\left(3 \mathrm{H}, \mathrm{d}, J 6.0, \mathrm{C}\left(4^{\prime}\right) \mathrm{CH}_{3}\right)$, $1.01\left(9 \mathrm{H}, \mathrm{s}, \mathrm{C}\left(\mathrm{CH}_{3}\right)_{3}\right), 0.99\left(3 \mathrm{H}, \mathrm{d}, J 6.2, \mathrm{C}\left(2^{\prime}\right) \mathrm{CH}_{3}\right) ;{ }^{13} \mathrm{C}\left\{{ }^{1} \mathrm{H}\right\}$ NMR (125 MHz, $\left.\mathrm{C}_{6} \mathrm{D}_{6}\right)$ major isomer 8d"', $198.9(C(7)), 179.4$ $(C(5)), 167.7\left(C_{2} \mathrm{CH}_{3}\right), 145.3\left(C\left(13^{\prime}\right)\right), 129.0\left(C\left(9^{\prime}\right)\right), 128.4$ $\left(C\left(11^{\prime}\right)\right), 118.2\left(C\left(10^{\prime}\right)\right), 118.0\left(C\left(8^{\prime}\right)\right), 113.1\left(C\left(12^{\prime}\right)\right), 100.3$ $(C(3)), 78.7(C(7 \mathrm{a})), 73.6\left(C\left(4^{\prime}\right)\right), 70.9\left(C\left(2^{\prime}\right)\right), 70.1(C(1)), 63.7$ $\left(C\left(5^{\prime}\right)\right), 58.0(C(6)), 52.8\left(C\left(1^{\prime}\right)\right), 52.7\left(\mathrm{CO}_{2} \mathrm{CH}_{3}\right), 37.6\left(\mathrm{C}\left(7^{\prime}\right)\right)$, $35.7 \quad\left(\mathrm{C}\left(\mathrm{CH}_{3}\right)_{3}\right), \quad 25.2 \quad\left(\mathrm{C}\left(\mathrm{CH}_{3}\right)_{3}\right), \quad 20.3 \quad\left(\mathrm{C}\left(4^{\prime}\right) \mathrm{CH}_{3}\right), \quad 19.0$ $\left(\mathrm{C}\left(2^{\prime}\right) C \mathrm{CH}_{3}\right) ; \mathrm{m} / \mathrm{z}\left(\mathrm{ESI}^{+}\right) 457.2\left(\mathrm{MH}^{+}, 100 \%\right) ; \mathrm{HRMS}\left(\mathrm{ESI}^{+}\right) \mathrm{m} / \mathrm{z}$ : $[\mathrm{MH}]+$ Calcd for $\mathrm{C}_{25} \mathrm{H}_{33} \mathrm{~N}_{2} \mathrm{O}_{6}$ 457.2333; Found 457.2324. Single Crystal Data: $\mathrm{C}_{25} \mathrm{H}_{32} \mathrm{~N}_{2} \mathrm{O}_{6}$, orthorhombic, $\mathrm{P} 2{ }_{1}{ }_{1} 2_{1}, a=9.0534(1)$, $b=11.9701(2), \quad c=22.0969(3) \AA, \quad V=2394.64(6) \AA^{3}$,
Data/restraints/parameters $\quad 4966 / 0 / 299, \quad \mathrm{R}_{\mathrm{int}}=0.039, \quad$ Flack $x=-0.07(12)$, Final $\mathrm{R}_{1}=0.0313, \mathrm{wR}_{2}=0.0805(\mathrm{I}>2 \mathrm{~s}(\mathrm{I}))$.

Methyl (2' $\left.S, 3 R, 4 ' R, 4 a^{\prime} R, 6 R, 7 a R\right)-3$-(tert-butyl)-2',4'-dimethyl5,7-dioxo-1',2',4',4a'-tetrahydro-1H,3H,5H,6' $\mathrm{H}$ spiro[pyrrolo[1,2-c] thiazole-6,5'-[1,4] oxazino[4,3a]quinoline]-7a(7H)-carboxylate (major isomer of $8 \mathrm{e}$ )

General procedure B $(8 \mathrm{~h})$ from tetramate $\mathbf{5 b}(250 \mathrm{mg}, 0.92 \mathrm{mmol})$ and $o$-aminobenzaldehyde $\mathbf{6 d}(222 \mathrm{mg}, 1.02 \mathrm{mmol})$ to give spirocycle 8e (111 mg, $0.235 \mathrm{mmol}, 23 \%)$ as an orange solid. m.p. $195-197{ }^{\circ} \mathrm{C} ; \mathrm{R}_{\mathrm{f}}\left(50 \%\right.$ EtOAc in petrol) $0.76 ; v_{\max } / \mathrm{cm}^{-1} 1773$, 1746, 1715; ${ }^{1} \mathrm{H}$ NMR $\left(500 \mathrm{MHz}, \mathrm{C}_{6} \mathrm{D}_{6}, 8: 2\right.$ mixture of diastereomers) major isomer 8e"', $7.14-7.10\left(1 \mathrm{H}, \mathrm{m}, \mathrm{C}\left(11^{\prime}\right) H\right)$, $6.72\left(1 \mathrm{H}, \mathrm{td}, J 7.3,0.9, \mathrm{C}\left(10^{\prime}\right) H\right), 6.68\left(1 \mathrm{H}, \mathrm{d}, J 7.3, \mathrm{C}\left(9^{\prime}\right) H\right), 6.59$ $\left(1 \mathrm{H}, \mathrm{d}, J 8.4, \mathrm{C}\left(12^{\prime}\right) H\right), 5.46(1 \mathrm{H}, \mathrm{s}, \mathrm{C}(3) H), 3.69-3.63(1 \mathrm{H}, \mathrm{m}$, $\left.\mathrm{C}\left(4^{\prime}\right) H\right), 3.63\left(1 \mathrm{H}, \mathrm{d}, J 11.4, \mathrm{C}(1) H_{\mathrm{A}} \mathrm{H}_{B}\right), 3.53-3.47(1 \mathrm{H}, \mathrm{m}$, $\left.\mathrm{C}\left(2^{\prime}\right) H\right), 3.44\left(1 \mathrm{H}, \mathrm{dd}, J 12.1,12.7, \mathrm{C}\left(1^{\prime}\right) H_{\mathrm{A}} \mathrm{H}_{\mathrm{B}}\right), 3.35(1 \mathrm{H}, \mathrm{d}, J$ 9.4, C(5')H), $3.30\left(1 \mathrm{H}, \mathrm{d}, J 16.4, \mathrm{C}\left(7^{\prime}\right) H_{\mathrm{A}} \mathrm{H}_{\mathrm{B}}\right), 3.18\left(3 \mathrm{H}, \mathrm{s}, \mathrm{OCH}_{3}\right)$, $2.51\left(1 \mathrm{H}, \mathrm{d}, J 11.5, \mathrm{C}(1) \mathrm{H}_{\mathrm{A}} H_{B}\right), 2.42(1 \mathrm{H}, \mathrm{dd}, J 12.1,10.5$, $\left.\mathrm{C}\left(1^{\prime}\right) \mathrm{H}_{\mathrm{A}} H_{B}\right), 2.33\left(1 \mathrm{H}, \mathrm{d}, J 16.4, \mathrm{C}\left(7^{\prime}\right) \mathrm{H}_{\mathrm{A}} H_{B}\right), 1.68(3 \mathrm{H}, \mathrm{d}, J 6.0$, $\left.\mathrm{C}\left(4^{\prime}\right) \mathrm{CH}_{3}\right), 0.98\left(9 \mathrm{H}, \mathrm{s}, \mathrm{C}\left(\mathrm{CH}_{3}\right)_{3}\right), 1.00-0.97\left(3 \mathrm{H}, \mathrm{m}, \mathrm{C}\left(2^{\prime}\right) \mathrm{CH}_{3}\right)$; ${ }^{13} \mathrm{C}\left\{{ }^{1} \mathrm{H}\right\}$ NMR $\left(125 \mathrm{MHz}, \mathrm{C}_{6} \mathrm{D}_{6}\right)$ major isomer 8e" ${ }^{\prime \prime \prime} 198.5(C(7))$, $179.1(C(5)), 167.4\left(C_{2} \mathrm{CH}_{3}\right), 145.0\left(C\left(13^{\prime}\right)\right), 129.1\left(C\left(9^{\prime}\right)\right)$, $128.5\left(C\left(11^{\prime}\right)\right), 118.3\left(C\left(10^{\prime}\right)\right), 118.1\left(C\left(8^{\prime}\right)\right), 113.3\left(C\left(12^{\prime}\right)\right), 82.9$ $(C(7 \mathrm{a})), 76.2(C(3)), 73.8\left(C\left(4^{\prime}\right)\right), 71.2\left(C\left(2^{\prime}\right)\right), 63.7\left(C\left(5^{\prime}\right)\right), 53.2$ $\left(C\left(1^{\prime}\right)\right), 53.0\left(\mathrm{CO}_{2} \mathrm{CH}_{3}\right), 51.9(C(6)), 39.1\left(C\left(7^{\prime}\right)\right), 37.4\left(C\left(\mathrm{CH}_{3}\right)_{3}\right)$, $\left.36.3(C(1)), 27.1\left(\mathrm{C}^{(} \mathrm{CH}_{3}\right)_{3}\right), 20.4\left(C\left(4^{\prime}\right) C \mathrm{H}_{3}\right), 19.1\left(\mathrm{C}\left(2^{\prime}\right) C \mathrm{H}_{3}\right)$; $\mathrm{m} / \mathrm{z}\left(\mathrm{ESI}^{+}\right) 473.2\left(\mathrm{MH}^{+}, 100 \%\right)$; HRMS $\left(\mathrm{ESI}^{+}\right) \mathrm{m} / \mathrm{z}:[\mathrm{MH}]+$ Calcd for $\mathrm{C}_{25} \mathrm{H}_{33} \mathrm{~N}_{2} \mathrm{O}_{5} \mathrm{~S}$ 473.2105; Found 473.2098. Single Crystal Data: $\mathrm{C}_{25} \mathrm{H}_{32} \mathrm{~N}_{2} \mathrm{O}_{6} \mathrm{~S}_{1}, \quad$ orthorhombic, $\quad \mathrm{P} 2{ }_{1} 2_{1} 2_{1}, \quad a=9.2640(2)$, $b=12.1677(3), \quad c=21.5109(6) \AA, \quad V=\quad 2424.74(10) \AA^{3}$, Data/restraints/parameters $\quad 5020 / 0 / 299, \quad \mathrm{R}_{\text {int }}=0.019$, Flack $x=-0.015(14)$, Final $\mathrm{R}_{1}=0.0320, \mathrm{wR}_{2}=0.0879(\mathrm{I}>2 \mathrm{~s}(\mathrm{I}))$.

Methyl (2' $\left.S, 3 R, 4^{\prime} R, 4 \mathrm{a}^{\prime} R, 6 R, 7 \mathrm{a} R\right)$-3-(tert-butyl)-2',4'-dimethyl8'-nitro-5,7-dioxo-1',2',4',4a'-tetrahydro-1H,3H,5H,6' $\mathrm{H}$ spiro[pyrrolo[1,2-c] oxazole-6,5'-[1,4] oxazino[4,3a]quinoline]-7a(7H)-carboxylate $(8 f)$

General procedure B $(15 \mathrm{~h})$ from tetramate $5 \mathbf{a}(70 \mathrm{mg}, 0.28 \mathrm{mmol})$ and $o$-aminobenzaldehyde $\mathbf{6 e}(86 \mathrm{mg}, 0.33 \mathrm{mmol})$ to give spirocycle $\mathbf{8 f}$ (4 mg, $0.008 \mathrm{mmol}, 3 \%)$ as an orange solid. m.p. 235 $-240{ }^{\circ} \mathrm{C} ; \mathrm{R}_{\mathrm{f}}\left(25 \%\right.$ EtOAc in petrol) $0.24 ; v_{\max } / \mathrm{cm}^{-1} 1745,1674$ and $1607 ;{ }^{1} \mathrm{H}$ NMR $\left(500 \mathrm{MHz}, \mathrm{C}_{6} \mathrm{D}_{6}, \quad 9: 1\right.$ mixture of diastereomers) major isomer 8f'"," $8.02(1 \mathrm{H}, \mathrm{dd}, J$ 9.3, 2.7, $\left.\mathrm{C}\left(11^{\prime}\right) H\right), 7.74\left(1 \mathrm{H}, \mathrm{d}, J 2.7, \mathrm{C}\left(9^{\prime}\right) H\right), 6.04\left(1 \mathrm{H}, \mathrm{d}, J 9.3, \mathrm{C}\left(12^{\prime}\right) H\right)$, $5.04(1 \mathrm{H}, \mathrm{s}, \mathrm{C}(3) H), 4.61\left(1 \mathrm{H}, \mathrm{d}, J 8.9, \mathrm{C}(1) H_{\mathrm{A}} \mathrm{H}_{\mathrm{B}}\right), 3.56(1 \mathrm{H}, \mathrm{dq}$, $J$ 9.6, 5.9, C(4')H), $3.25-3.19\left(1 \mathrm{H}, \mathrm{m}, \mathrm{C}\left(2^{\prime}\right) H\right), 3.16(3 \mathrm{H}, \mathrm{s}$, $\left.\mathrm{CO}_{2} \mathrm{CH}_{3}\right), 3.18-3.14\left(1 \mathrm{H}, \mathrm{m}, \mathrm{C}\left(5^{\prime}\right) H\right), 3.09(1 \mathrm{H}, \mathrm{dd}, J$ 12.5, 2.7, $\left.\mathrm{C}\left(1^{\prime}\right) H_{\mathrm{A}} \mathrm{H}_{\mathrm{B}}\right), 2.96\left(1 \mathrm{H}, \mathrm{d}, \mathrm{J} 9.0, \mathrm{C}(1) \mathrm{H}_{\mathrm{A}} H_{B}\right), 2.86(1 \mathrm{H}, \mathrm{d}, J 16.5$, $\left.\mathrm{C}\left(7^{\prime}\right) H_{\mathrm{A}} \mathrm{H}_{\mathrm{B}}\right), 2.21\left(1 \mathrm{H}, \mathrm{dd}, J 12.5,10.8, \mathrm{C}\left(1^{\prime}\right) \mathrm{H}_{\mathrm{A}} H_{B}\right), 2.02(1 \mathrm{H}, \mathrm{d}$, $J$ 16.7, $\left.\mathrm{C}\left(7^{\prime}\right) \mathrm{H}_{\mathrm{A}} H_{B}\right), 1.61\left(3 \mathrm{H}, \mathrm{d}, J 6.0, \mathrm{C}\left(4^{\prime}\right) \mathrm{CH}_{3}\right), 0.99(9 \mathrm{H}, \mathrm{s}$, $\left.\mathrm{C}\left(\mathrm{CH}_{3}\right)_{3}\right), 0.91\left(3 \mathrm{H}, \mathrm{d}, J 6.2, \mathrm{C}\left(2^{\prime}\right) \mathrm{CH}_{3}\right) ;{ }^{13} \mathrm{C}\left\{{ }^{1} \mathrm{H}\right\} \mathrm{NMR}(125 \mathrm{MHz}$, $\left.\mathrm{C}_{6} \mathrm{D}_{6}\right)$ major isomer 8f'", $198.9(C(7)), 178.0(C(5)), 167.3$ $\left(\mathrm{CO}_{2} \mathrm{CH}_{3}\right), 149.3\left(C\left(13^{\prime}\right)\right), 138.7\left(C\left(10^{\prime}\right)\right), 124.8$ (C(9')), 124.4 $\left(C\left(11^{\prime}\right)\right), 117.3\left(C\left(8^{\prime}\right)\right), 111.3\left(C\left(12^{\prime}\right)\right), 100.2(C(3)), 78.4(C(7 \mathrm{a}))$, $73.0\left(C\left(4^{\prime}\right)\right), \quad 70.3\left(C\left(2^{\prime}\right)\right), 69.8(C(1)), 62.9\left(C\left(5^{\prime}\right)\right), 52.7$ $\left(\mathrm{CO}_{2} \mathrm{CH}_{3}\right), 51.8\left(C\left(1^{\prime}\right)\right), 51.8(C(6)), 36.5\left(C\left(7^{\prime}\right)\right), 35.5\left(C\left(\mathrm{CH}_{3}\right)_{3}\right)$, $24.9\left(\mathrm{C}\left(\mathrm{CH}_{3}\right)_{3}\right), 20.0\left(\mathrm{C}\left(4^{\prime}\right) C \mathrm{H}_{3}\right), 18.6\left(\mathrm{C}\left(2^{\prime}\right) C \mathrm{H}_{3}\right) ; \mathrm{m} / \mathrm{z}\left(\mathrm{ESI}^{+}\right)$ $502.3\left(\mathrm{MH}^{+}, 73 \%\right)$; HRMS $\left(\mathrm{ESI}^{+}\right) \mathrm{m} / \mathrm{z}:[\mathrm{MH}]+$ Calcd for $\mathrm{C}_{25} \mathrm{H}_{32} \mathrm{~N}_{3} \mathrm{O}_{8}$ 502.2184; Found 502.2186.

Methyl (2' $\left.S, 3 R, 4^{\prime} R, 4 \mathrm{a}^{\prime} R, 6 R, 7 \mathrm{a} R\right)$-3-(tert-butyl)-2',4'-dimethyl8 '-nitro-5,7-dioxo-1',2',4',4a'-tetrahydro-1H,3H,5H,6' $\mathrm{H}$ spiro[pyrrolo[1,2-c] thiazole-6,5'-[1,4] oxazino[4,3a]quinoline]-7a(7H)-carboxylate $(8 \mathrm{~g})$ 
General procedure B $(8 \mathrm{~h})$ from tetramate $\mathbf{5 b}(75 \mathrm{mg}, 0.28 \mathrm{mmol})$ and $o$-aminobenzaldehyde $6 \mathrm{e}(88 \mathrm{mg}, 0.33 \mathrm{mmol})$ to give spirocycle $\mathbf{8 g}$ (13 mg, $0.024 \mathrm{mmol}, 9 \%$ ) as an orange solid. m.p. $236-238{ }^{\circ} \mathrm{C} ; \mathrm{R}_{\mathrm{f}}\left(25 \%\right.$ EtOAc in petrol) $0.41 ; v_{\max } / \mathrm{cm}^{-1} 1744$, $1718,1685,1316 ;{ }^{1} \mathrm{H}$ NMR $\left(500 \mathrm{MHz}, \mathrm{C}_{6} \mathrm{D}_{6}, 8: 1\right.$ mixture of diastereomers) major isomer $\mathbf{8 g}, ", \quad 8.01(1 \mathrm{H}, \mathrm{dd}, J$ 9.3, 2.8, $\left.\mathrm{C}\left(11^{\prime}\right) H\right), 7.63\left(1 \mathrm{H}, \mathrm{d}, J 2.7, \mathrm{C}\left(9^{\prime}\right) H\right), 6.03\left(1 \mathrm{H}, \mathrm{d}, J 9.3, \mathrm{C}\left(12^{\prime}\right) H\right)$, $5.41(1 \mathrm{H}, \mathrm{s}, \mathrm{C}(3) H), 3.55\left(1 \mathrm{H}, \mathrm{d}, J 11.6, \mathrm{C}(1) H_{\mathrm{A}} \mathrm{H}_{\mathrm{B}}\right), 3.53-3.48$ $\left(1 \mathrm{H}, \mathrm{m}, \mathrm{C}\left(4^{\prime}\right) H\right), 3.21\left(1 \mathrm{H}, \mathrm{d}, J 9.6, \mathrm{C}\left(5^{\prime}\right) H\right), 3.24-3.19(1 \mathrm{H}, \mathrm{m}$, $\left.\mathrm{C}\left(2^{\prime}\right) H\right), 3.16\left(3 \mathrm{H}, \mathrm{s}, \mathrm{CO}_{2} \mathrm{CH}_{3}\right), 3.15-3.09\left(1 \mathrm{H}, \mathrm{m}, \mathrm{C}\left(1^{\prime}\right) \mathrm{H}_{\mathrm{A}} \mathrm{H}_{\mathrm{B}}\right)$, $2.85\left(1 \mathrm{H}, \mathrm{d}, J 16.4, \mathrm{C}\left(7^{\prime}\right) H_{\mathrm{A}} \mathrm{H}_{\mathrm{B}}\right), 2.39\left(1 \mathrm{H}, \mathrm{d}, J 11.5, \mathrm{C}(1) \mathrm{H}_{\mathrm{A}} H_{B}\right)$, $2.21\left(1 \mathrm{H}\right.$, dd. $\left.J 12.6,10.7, \mathrm{C}\left(1^{\prime}\right) \mathrm{H}_{\mathrm{A}} H_{B}\right), 1.96(1 \mathrm{H}, \mathrm{d}, J 16.5$, $\left.\mathrm{C}\left(7^{\prime}\right) \mathrm{H}_{\mathrm{A}} H_{B}\right), 1.60\left(3 \mathrm{H}, \mathrm{d}, J 6.0, \mathrm{C}\left(4^{\prime}\right) \mathrm{CH}_{3}\right), 0.97\left(9 \mathrm{H}, \mathrm{s}, \mathrm{C}\left(\mathrm{CH}_{3}\right)_{3}\right)$, $0.91\left(3 \mathrm{H}, \mathrm{d}, J 6.2, \mathrm{C}\left(2^{\prime}\right) \mathrm{CH}_{3}\right) ;{ }^{13} \mathrm{C}\left\{{ }^{1} \mathrm{H}\right\}$ NMR $\left(125 \mathrm{MHz}, \mathrm{C}_{6} \mathrm{D}_{6}\right)$ major isomer 8g'",' $200.1(C(7)), 176.3(C(5)), 167.8\left(C_{2} \mathrm{CH}_{3}\right)$, $149.7\left(C\left(13^{\prime}\right)\right), 139.0\left(C\left(10^{\prime}\right)\right), 125.2\left(C\left(9^{\prime}\right)\right), 124.9\left(C\left(11^{\prime}\right)\right)$, $117.6\left(C\left(8^{\prime}\right)\right), 111.6\left(C\left(12^{\prime}\right)\right), 82.7(C(7 \mathrm{a})), 76.1(C(3)), 73.4$ $\left(C\left(4^{\prime}\right)\right), 70.7\left(C\left(2^{\prime}\right)\right), 63.2\left(C\left(5^{\prime}\right)\right), 53.1\left(\mathrm{CO}_{2} \mathrm{CH}_{3}\right), 52.3\left(C\left(1^{\prime}\right)\right)$, $51.1(C(6)), 38.0\left(C\left(7^{\prime}\right)\right), 37.4\left(C\left(\mathrm{CH}_{3}\right)_{3}\right), 36.2(C(1)), 27.0$ $\left.\left.\left(\mathrm{C}^{(} \mathrm{CH}_{3}\right)_{3}\right), 20.3\left(\mathrm{C}^{\prime} 4^{\prime}\right) \mathrm{CH}_{3}\right), 18.9\left(\mathrm{C}\left(2^{\prime}\right) \mathrm{CH}_{3}\right)$; HRMS (APCI $\left.{ }^{+}\right)$ $\mathrm{m} / \mathrm{z}$ : $[\mathrm{MH}]+$ Calcd for $\mathrm{C}_{25} \mathrm{H}_{32} \mathrm{~N}_{3} \mathrm{O}_{7} \mathrm{~S}$ 518.1956; Found 518.1959.

\section{Methyl (3'R,7a'R)-7-bromo-3'-(tert-butyl)-5',7'-dioxo-1,2,3,3a- tetrahydro-1' $H, 3{ }^{\prime} H, 5 H, 5^{\prime} H$-spiro[pyrrolo[1,2-a]quinoline- 4,6'-pyrrolo[1,2-c] oxazole $]-7 \mathbf{a}^{\prime}\left(7^{\prime} H\right)$-carboxylate $(8 \mathbf{i})$}

General procedure B $(6 \mathrm{~h})$ from tetramate 5a $(100 \mathrm{mg}, 0.392$ $\mathrm{mmol})$ and $o$-aminobenzaldehyde $23(110 \mathrm{mg}, 0.431 \mathrm{mmol})$ to give spirocycle $8 \mathbf{i}(119 \mathrm{mg}, 0.243 \mathrm{mmol}, 9 \%)$ as a brown solid. $\mathrm{R}_{\mathrm{f}}$ (25\% EtOAc in petrol) $0.81 ; v_{\max } / \mathrm{cm}^{-1} 1742,1718,1112 ;{ }^{1} \mathrm{H}$ NMR $\left(500 \mathrm{MHz}, \mathrm{C}_{6} \mathrm{D}_{6}, 2.5: 1.5: 1: 1\right.$ mixture of 4 diastereomers) major isomer $7.26\left(1 \mathrm{H}, \mathrm{dd}, J\right.$ 8.6, 2.7, $\left.\mathrm{C}\left(11^{\prime}\right) H\right), 7.08(1 \mathrm{H}, \mathrm{d}, J 2.3$, $\left.\mathrm{C}\left(9^{\prime}\right) H\right), 6.12(1 \mathrm{H}, \mathrm{d}, J$ 8.6, C(12')H $), 5.11(1 \mathrm{H}, \mathrm{s}, \mathrm{C}(3) H), 4.74$ $\left(1 \mathrm{H}, \mathrm{d}, J 8.9, \mathrm{C}(1) H_{\mathrm{A}} \mathrm{H}_{\mathrm{B}}\right), 3.67\left(1 \mathrm{H}, \mathrm{dd}, J 10.3,5.6, \mathrm{C}\left(5^{\prime}\right) H\right), 3.28$ $\left(1 \mathrm{H}, \mathrm{d}, J 16.1, \mathrm{C}\left(7^{\prime}\right) H_{\mathrm{A}} \mathrm{H}_{\mathrm{B}}\right), 3.14\left(1 \mathrm{H}, \mathrm{d}, J 8.9, \mathrm{C}(1) \mathrm{H}_{\mathrm{A}} H_{\mathrm{B}}\right), 3.05$ $\left(1 \mathrm{H}, \mathrm{d}, J 16.3, \mathrm{C}\left(7^{\prime}\right) \mathrm{H}_{\mathrm{A}} H_{\mathrm{B}}\right), 3.04\left(3 \mathrm{H}, \mathrm{s}, \mathrm{CO}_{2} \mathrm{CH}_{3}\right), 2.91(1 \mathrm{H}, \mathrm{ddd}$, $J$ 8.2, 5.1, 2.9, C(1') $\left.H_{\mathrm{A}} \mathrm{H}_{\mathrm{B}}\right), 2.82-2.74\left(1 \mathrm{H}, \mathrm{m}, \mathrm{C}\left(1^{\prime}\right) \mathrm{H}_{\mathrm{A}} H_{\mathrm{B}}\right), 1.69$ $-1.60\left(1 \mathrm{H}, \mathrm{m}, \mathrm{C}\left(4^{\prime}\right) H_{\mathrm{A}} \mathrm{H}_{\mathrm{B}}\right), 1.49\left(1 \mathrm{H}, \mathrm{m}, \mathrm{C}\left(2^{\prime}\right) H_{\mathrm{A}} \mathrm{H}_{\mathrm{B}}\right), 1.43-1.38$ $\left(1 \mathrm{H}, \mathrm{m}, \mathrm{C}\left(2^{\prime}\right) \mathrm{H}_{\mathrm{A}} H_{\mathrm{B}}\right), 1.01\left(9 \mathrm{H}, \mathrm{s}, \mathrm{C}\left(\mathrm{CH}_{3}\right)_{3}\right), 0.87-0.81(1 \mathrm{H}, \mathrm{m}$, $\left.\mathrm{C}\left(4^{\prime}\right) \mathrm{H}_{\mathrm{A}} H_{\mathrm{B}}\right) ;{ }^{13} \mathrm{C}\left\{{ }^{1} \mathrm{H}\right\}$ NMR $\left(125 \mathrm{MHz}, \mathrm{C}_{6} \mathrm{D}_{6}\right)$ major isomer 199.8 $(C(7)), 178.4(C(5)), 167.2\left(C_{2} \mathrm{CH}_{3}\right), 143.1\left(C\left(13^{\prime}\right)\right), 131.3$ (C(9')), $131.2\left(C\left(11^{\prime}\right)\right), 117.9\left(C\left(8^{\prime}\right)\right), 112.8\left(C\left(12^{\prime}\right)\right), 107.8$ $\left(C\left(10^{\prime}\right)\right), 99.0(C(3)), 78.4(C(7 \mathrm{a})), 68.0(C(1)), 60.4\left(C\left(5^{\prime}\right)\right), 53.1$ $\left(\mathrm{CO}_{2} \mathrm{CH}_{3}\right), 52.3(\mathrm{C}(6)), 47.6\left(\mathrm{C}\left(1^{\prime}\right)\right), 37.3\left(\mathrm{C}\left(7^{\prime}\right)\right), 35.7\left(\mathrm{C}\left(\mathrm{CH}_{3}\right)_{3}\right)$, $28.9\left(C\left(4^{\prime}\right)\right), 25.0\left(\mathrm{C}\left(\mathrm{CH}_{3}\right)_{3}\right), 23.8\left(C\left(2^{\prime}\right)\right) ; \mathrm{m} / z\left(\mathrm{ESI}^{+}\right) 491.2\left(\mathrm{MH}^{+}\right.$, $100 \%)$ HRMS $\left(\mathrm{ESI}^{+}\right) \mathrm{m} / \mathrm{z}:[\mathrm{MH}]+$ Calcd for $\mathrm{C}_{23} \mathrm{H}_{28} \mathrm{BrN}_{2} \mathrm{O}_{5}$ 491.1176; Found 491.1174.

\section{Methyl (3'R,7a'R)-3' -(tert-butyl)-5',7'-dioxo-7-phenyl- 1,2,3,3a-tetrahydro-1' $H, 3 ' H, 5 H, 5 ' H$-spiro[pyrrolo[1,2- a]quinoline-4,6'-pyrrolo[1,2-c]oxazole $]-7 a^{\prime}\left(7^{\prime} H\right)$-carboxylate (8j)}

Bromospirocycle 8i (100 mg, $0.20 \mathrm{mmol}$ ) and phenylboronic acid (37 mg, $0.30 \mathrm{mmol})$ were dissolved into THF $(5 \mathrm{~mL})$. To the stirring solution was added $\mathrm{Pd}(\mathrm{dppf}) \mathrm{Cl}_{2}\left(195 \mathrm{mg}\right.$ in $0.2 \mathrm{~mL} \mathrm{H}_{2} \mathrm{O}$, $0.01 \mathrm{mmol}, 5 \mathrm{~mol} \%$ ). The mixture was stirred at room temperature for 40 minutes and then heated to reflux for $24 \mathrm{~h}$. The reaction mixture was then diluted with EtOAc and filtered through a pad of Celite. The filtrates were collected and washed with brine and a saturated solution of $\mathrm{NaHCO}_{3}$. Solvent was removed using rotary evaporation and the resulting residue was purified by flash column chromatography to yield the biaryl $\mathbf{8 j}$ ( $38 \mathrm{mg}, 0.078 \mathrm{mmol}, 39 \%$ ) as a mixture of 4 diastereomers as a yellow oil. $\mathrm{R}_{\mathrm{f}}(25 \%$ EtOAc in petrol) $0.91 ; v_{\max } / \mathrm{cm}^{-1} 1745,1711,1605,1507,1480,1461,1577$; $\mathrm{m} / \mathrm{z}\left(\mathrm{ESI}^{+}\right) 489.2\left(\mathrm{MH}^{+}, 100 \%\right) ; \mathrm{HRMS}\left(\mathrm{ESI}^{+}\right) \mathrm{m} / \mathrm{z}:[\mathrm{MH}]+$ Calcd for $\mathrm{C}_{29} \mathrm{H}_{33} \mathrm{~N}_{2} \mathrm{O}_{5}$ 489. 2384; Found 489.2382.

\section{Methyl (3R,7aR)-6-benzylidene-3-(tert-butyl)-5,7- dioxodihydro-1 $H, 3 H$-pyrrolo[1,2-c]oxazole-7a $(5 H)$ - carboxylate (10)}

Tetramare 5a (100 mg, $0.392 \mathrm{mmol})$ was added to a solution of benzaldehyde $(440 \mu \mathrm{L}, 0.429 \mathrm{mmol})$ in methanol $(2.0 \mathrm{~mL})$. The solution was then stirred at room temperature for 30 hours, and then concentrated in vacuo. Purification via flash column chromatography gave alkene $\mathbf{1 0}(47 \mathrm{mg}, 0.14 \mathrm{mmol}, 36 \%, 1.2: 1$ mixture of E/Z isomers) as a yellow oil. $R_{\mathrm{f}}\left(\right.$ EtOAc) $0.41 ; v_{\max } / \mathrm{cm}^{-}$ ${ }^{1} 1700,1622,1475 ;{ }^{1} \mathrm{H}$ NMR $\left(500 \mathrm{MHz}, \mathrm{CDCl}_{3}\right) E$ isomer: $8.35-$ $8.32\left(2 \mathrm{H}, \mathrm{m}, \mathrm{C}\left(3^{\prime}\right) H\right), 7.92\left(1 \mathrm{H}, \mathrm{s}, \mathrm{C}\left(1^{\prime}\right) H\right), 7.63-7.56(1 \mathrm{H}, \mathrm{m}$, $\left.\mathrm{C}\left(5^{\prime}\right) H\right), 7.53-7.48\left(2 \mathrm{H}, \mathrm{m}, \mathrm{C}\left(4^{\prime}\right) H\right), 5.07(1 \mathrm{H}, \mathrm{s}, \mathrm{C}(3) H), 4.90$ $\left(1 \mathrm{H}, \mathrm{d}, J 9.0, \mathrm{C}(1) H_{\mathrm{A}} \mathrm{H}_{\mathrm{B}}\right), 3.78\left(3 \mathrm{H}, \mathrm{s}, \mathrm{CO}_{2} \mathrm{CH}_{3}\right), 3.57(1 \mathrm{H}, \mathrm{d}, J 9.0$, $\left.\mathrm{C}(1) \mathrm{H}_{\mathrm{A}} H_{B}\right), 0.97\left(9 \mathrm{H}, \mathrm{s}, \mathrm{C}\left(\mathrm{CH}_{3}\right)_{3}\right)$; $\mathrm{Z}$ isomer: $8.45-8.41(2 \mathrm{H}, \mathrm{m}$, $\left.\mathrm{C}\left(3^{\prime}\right) H\right), 7.82\left(1 \mathrm{H}, \mathrm{s}, \mathrm{C}\left(1^{\prime}\right) H\right), 7.63-7.56\left(1 \mathrm{H}, \mathrm{m}, \mathrm{C}\left(5^{\prime}\right) H\right), 7.53$ - $7.48\left(2 \mathrm{H}, \mathrm{m}, \mathrm{C}\left(4^{\prime}\right) H\right), 5.09(1 \mathrm{H}, \mathrm{s}, \mathrm{C}(3) H), 4.90(1 \mathrm{H}, \mathrm{d}, J 8.5$, $\left.\mathrm{C}(1) H_{\mathrm{A}} \mathrm{H}_{\mathrm{B}}\right), 3.79\left(3 \mathrm{H}, \mathrm{s}, \mathrm{CO}_{2} \mathrm{CH}_{3}\right), 3.58\left(1 \mathrm{H}, \mathrm{d}, J 8.5, \mathrm{C}(1) \mathrm{H}_{\mathrm{A}} H_{B}\right)$, $0.97\left(9 \mathrm{H}, \mathrm{s},\left(\mathrm{CH}_{3}\right)_{3}\right) ;{ }^{13} \mathrm{C}\left\{{ }^{1} \mathrm{H}\right\} \mathrm{NMR}\left(125 \mathrm{MHz}, \mathrm{CDCl}_{3}\right) E$ isomer: $190.0(C(7)), 172.1(C(5)), 167.8\left(C_{2} \mathrm{CH}_{3}\right), 154.1\left(C\left(1^{\prime}\right)\right), 134.9$ $\left(C\left(5^{\prime}\right)\right), 134.4\left(C\left(3^{\prime}\right)\right), 132.9\left(C\left(2^{\prime}\right)\right), 129.1\left(C\left(4^{\prime}\right)\right), 123.3(C(6))$, $99.3(\mathrm{C}(3)), 77.1(\mathrm{C}(7 \mathrm{a})), 68.6(\mathrm{C}(1)), 53.6\left(\mathrm{CO}_{2} \mathrm{CH}_{3}\right), 35.6$ $\left(C\left(\mathrm{CH}_{3}\right)_{3}\right), 24.9\left(\mathrm{C}\left(\mathrm{CH}_{3}\right)_{3}\right) ; \mathrm{Z}$ isomer: $191.5(\mathrm{C}(7)), 170.3(\mathrm{C}(5))$, $167.6\left(\mathrm{CO}_{2} \mathrm{CH}_{3}\right), 152.2\left(C\left(1^{\prime}\right)\right), 135.4\left(C\left(3^{\prime}\right)\right), 134.5\left(C\left(5^{\prime}\right)\right), 132.3$ $\left(C\left(2^{\prime}\right)\right), 129.1\left(C\left(4^{\prime}\right)\right), 123.9(C(6)), 99.0(C(3)), 77.8(C(7 \mathrm{a})), 68.3$ $(\mathrm{C}(1)), 53.6\left(\mathrm{CO}_{2} \mathrm{CH}_{3}\right), 35.7\left(\mathrm{C}\left(\mathrm{CH}_{3}\right)_{3}\right), 25.0\left(\mathrm{C}\left(\mathrm{CH}_{3}\right)_{3}\right) ; \mathrm{m} / z\left(\mathrm{ESI}^{+}\right)$ $344.1\left(\mathrm{MH}^{+}, 100 \%\right)$; HRMS $\left(\mathrm{ESI}^{+}\right) \mathrm{m} / \mathrm{z}:[\mathrm{MH}]+$ Calcd for $\mathrm{C}_{19} \mathrm{H}_{22} \mathrm{NO}_{5}$ 344.1492; Found 344.1486.

Methyl

$(3 R, 7 \mathrm{a} R)-3-($ tert-butyl)-6-(2-(2,6dimethylmorpholino)benzyl)-7-hydroxy-5-oxo- $1 \mathrm{H}, 3 \mathrm{H}$ pyrrolo[1,2-c]oxazole-7a(5H)-carboxylate (14)

Acetic acid $(60 \mu \mathrm{L}, 0.99 \mathrm{mmol})$ and spirotetramate 8d (100 mg, $0.219 \mathrm{mmol})$ were dissolved in anhydrous DCM $(600 \mu \mathrm{L}) . \mathrm{NaBH}_{4}$ was added portionwise at $0{ }^{\circ} \mathrm{C}$ and left to stir for $15 \mathrm{~min}$ before stirring at room temperature. After $36 \mathrm{~h}$, the reaction mixture was quenched with saturated aqueous $\mathrm{NaHCO}_{3}$, extracted with EtOAc, washed with brine, dried over anhydrous $\mathrm{Na}_{2} \mathrm{SO}_{4}$ and concentrated in vacuo to give $14(56 \mathrm{mg}, 0.12 \mathrm{mmol}, 56 \%)$ as a pink oil. $\mathrm{R}_{\mathrm{f}}(20 \%$ EtOAc in petrol) $0.53 ; v_{\max } / \mathrm{cm}^{-1} ; 2976,1746,1716,1664 ;{ }^{1} \mathrm{H}$ NMR $\left(500 \mathrm{MHz}, \mathrm{C}_{6} \mathrm{D}_{6}\right) 7.49(1 \mathrm{H}, \mathrm{dd}, J$ 7.2, 2.1, C(9')H), $6.98-6.88$ $\left(2 \mathrm{H}, \mathrm{m}, \mathrm{C}\left(10^{\prime}\right) H+\mathrm{C}\left(11^{\prime}\right) H\right), 6.63\left(1 \mathrm{H}, \mathrm{dd}, J 7.6,1.7, \mathrm{C}\left(12^{\prime}\right) H\right)$, $4.92\left(1 \mathrm{H}, \mathrm{d}, J 8.3, \mathrm{C}(1) H_{\mathrm{A}} \mathrm{H}_{\mathrm{B}}\right), 4.90(1 \mathrm{H}, \mathrm{s}, \mathrm{C}(3) H), 3.82(1 \mathrm{H}, \mathrm{dqd}$, $J$ 10.6, 6.2, 2.0, C(2')H), $3.70\left(1 \mathrm{H}, \mathrm{dqd}, J 10.6,6.2,2.0, \mathrm{C}\left(2^{\prime}\right) H\right)$, $3.43\left(1 \mathrm{H}, \mathrm{d}, J 14.4, \mathrm{C}\left(7^{\prime}\right) H_{\mathrm{A}} \mathrm{H}_{\mathrm{B}}\right), 3.34\left(1 \mathrm{H}, \mathrm{d}, J 14.4, \mathrm{C}\left(7^{\prime}\right) \mathrm{H}_{\mathrm{A}} H_{B}\right)$, $3.30\left(3 \mathrm{H}, \mathrm{s}, \mathrm{CO}_{2} \mathrm{CH}_{3}\right), 3.08\left(1 \mathrm{H}, \mathrm{d}, J 8.3, \mathrm{C}(1) \mathrm{H}_{\mathrm{A}} H_{B}\right), 2.58-2.55$ $\left(1 \mathrm{H}, \mathrm{m}, \mathrm{C}\left(5^{\prime}\right) H_{\mathrm{A}} \mathrm{H}_{\mathrm{B}}\right), 2.54-2.50\left(1 \mathrm{H}, \mathrm{m}, \mathrm{C}\left(1^{\prime}\right) H_{\mathrm{A}} \mathrm{H}_{\mathrm{B}}\right), 2.15(1 \mathrm{H}$, t, $\left.J 11.0, \mathrm{C}\left(5^{\prime}\right) \mathrm{H}_{\mathrm{A}} H_{B}\right), 1.95\left(1 \mathrm{H}, \mathrm{t}, J 11.0, \mathrm{C}\left(1^{\prime}\right) \mathrm{H}_{\mathrm{A}} H_{B}\right), 1.14(9 \mathrm{H}$, s, $\left.\mathrm{C}\left(\mathrm{CH}_{3}\right)_{3}\right), 0.93\left(3 \mathrm{H}, \mathrm{d}, J 6.2, \mathrm{C}\left(4^{\prime}\right) \mathrm{CH}_{3}\right), 0.87(3 \mathrm{H}, \mathrm{d}, J 6.3$, $\left.\mathrm{C}\left(2^{\prime}\right) \mathrm{CH}_{3}\right) ;{ }^{13} \mathrm{C}\left\{{ }^{1} \mathrm{H}\right\}$ NMR $\left(125 \mathrm{MHz}, \mathrm{C}_{6} \mathrm{D}_{6}\right) 178.7(C(5)), 171.4$ $(C(7)), 169.5 \quad\left(\mathrm{CO}_{2} \mathrm{CH}_{3}\right), 147.3\left(C\left(12^{\prime}\right)\right), 134.8\left(C\left(8^{\prime}\right)\right), 132.7$

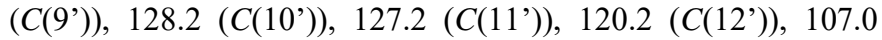
(C(6)), $97.2(C(3)), 74.2(C(7 \mathrm{a})), 70.6\left(C\left(2^{\prime}\right)\right), 70.6\left(C\left(4^{\prime}\right)\right), 70.2$ $(C(1)), 60.3\left(C\left(1^{\prime}\right)\right), 58.8\left(C\left(5^{\prime}\right)\right), 52.5\left(\mathrm{CO}_{2} C \mathrm{H}_{3}\right), 35.6\left(C\left(\mathrm{CH}_{3}\right)_{3}\right)$, $\left.25.2\left(\mathrm{C}\left(\mathrm{CH}_{3}\right)_{3}\right), 24.6\left(\mathrm{C}^{\prime} 7^{\prime}\right)\right), 19.2\left(\mathrm{C}\left(4^{\prime}\right) \mathrm{CH}_{3}\right), 19.1\left(\mathrm{C}\left(2^{\prime}\right) \mathrm{CH}_{3}\right)$; $\mathrm{m} / \mathrm{z}\left(\mathrm{ESI}^{+}\right) 459.3\left(\mathrm{MH}^{+}, 100 \%\right)$.

\section{(3S,7aR)-3-(2-Chloro-4-fluorophenyl)dihydro-3H,5H- pyrrolo[1,2-c] thiazole-5,7(6H)-dione (17)}

To a stirred solution of tetramate $16(185 \mathrm{mg}, 0.52 \mathrm{mmol})$ in MeCN (5 mL) was added one drop of distilled water, and the reaction mixture was heated to reflux for $6 \mathrm{~h}$. The solvent was removed in vacuo to yield tetramate $17(138 \mathrm{mg}, 0.483 \mathrm{mmol}$, 93\%) as a dark orange oil, which was used in the following step without further purification. $\mathrm{R}_{\mathrm{f}}(20 \% \mathrm{MeOH}$ in EtOAc) 0.15; $[\alpha]_{D}^{25}-257.0\left(c 0.25, \mathrm{CHCl}_{3}\right) ; v_{\max } / \mathrm{cm}^{-1} 2981,1696,1649,1232$, 
728; ${ }^{1} \mathrm{H}$ NMR $\left(400 \mathrm{MHz}, \mathrm{CDCl}_{3}\right) 7.29\left(1 \mathrm{H}, \mathrm{dd}, J 8.7,5.8, \mathrm{C}\left(6^{\prime}\right) H\right)$, 7.17 (1H, dd, $\left.J 8.2,2.6, \mathrm{C}\left(3^{\prime}\right) H\right), 6.99$ (1H, td, $J$ 8.2, 2.6, C(5')H), $6.74(1 \mathrm{H}, \mathrm{s}, \mathrm{C}(3) H), 4.82-4.73(1 \mathrm{H}, \mathrm{m}, \mathrm{C}(7 \mathrm{a}) H), 3.44(1 \mathrm{H}, \mathrm{d}, J$ $\left.22.1, \mathrm{C}(6) H_{\mathrm{A}} \mathrm{H}_{\mathrm{B}}\right), 3.32-3.22\left(2 \mathrm{H}, \mathrm{m}, \mathrm{C}(1) H_{\mathrm{A}} \mathrm{H}_{\mathrm{B}}+\mathrm{C}(6) \mathrm{H}_{\mathrm{A}} H_{B}\right)$, $3.09\left(1 \mathrm{H}, \mathrm{dd}, J=10.9,9.6 \mathrm{~Hz}, \mathrm{C}(1) \mathrm{H}_{\mathrm{A}} H_{B}\right) ;{ }^{13} \mathrm{C}\left\{{ }^{1} \mathrm{H}\right\} \mathrm{NMR}$ $\left(100 \mathrm{MHz}, \mathrm{CDCl}_{3}\right) 202.2$ (C(7)), $169.1(C(5)), 162.3$ (d, J 249.7, $\left.C\left(4^{\prime}\right)\right), 133.6\left(\mathrm{~d}, J 4.1, C\left(2^{\prime}\right)\right), 126.6\left(\mathrm{~d}, J 9.2, C\left(6^{\prime}\right)\right), 117.9(\mathrm{~d}, J$ 25.1, $\left.C\left(3^{\prime}\right)\right), 114.5\left(\mathrm{~d}, J 21.5, C\left(5^{\prime}\right)\right), 100.1\left(C\left(1^{\prime}\right)\right), 72.5(C(7 \mathrm{a}))$, $60.4(C(3)), 43.5(C(6)), 33.3(C(1)) ; m / z\left(\mathrm{ESI}^{-}\right) 284.0\left([\mathrm{M}-\mathrm{H}]^{-}\right.$, $100 \%$ ); HRMS (ESI-) m/z: [M-H] $]^{-}$Calcd for $\mathrm{C}_{12} \mathrm{H}_{8} \mathrm{ClFNO}_{2} \mathrm{~S}$ 283.9954; Found 283.9954

\section{2-(Diethylamino)benzaldehyde (21a)}

General procedure A $(24 \mathrm{~h})$ from 2 -fluorobenzaldehyde $(300 \mu \mathrm{L}$, $4.75 \mathrm{mmol})$, diethylamine $(565 \mu \mathrm{L}, 5.46 \mathrm{mmol})$ and potassium carbonate (755 mg, $5.46 \mathrm{mmol}$ ) to give 21a $(150 \mathrm{mg}, 0.846 \mathrm{mmol}$, $18 \%)$ as a brown oil. $\mathrm{R}_{\mathrm{f}}(20 \%$ hexane in DCM $) 0.48 ; v_{\max } / \mathrm{cm}^{-1}$ $1683,1594,1482,1450 ;{ }^{1} \mathrm{H}$ NMR $\left(400 \mathrm{MHz}, \mathrm{CDCl}_{3}\right) 10.4(1 \mathrm{H}, \mathrm{s}$, CHO), $7.80(1 \mathrm{H}, \mathrm{dd}, J 7.7,1.8, \mathrm{C}(6) H), 7.49(1 \mathrm{H}, \mathrm{ddd}, J 8.2,7.2$, $1.8, \mathrm{C}(4) H), 7.15(1 \mathrm{H}, \mathrm{d}, J 8.2, \mathrm{C}(3) H), 7.13-7.03(1 \mathrm{H}, \mathrm{m}$, $\mathrm{C}(5) H), 3.18(4 \mathrm{H}, \mathrm{q}, J$ 7.1, C(1')H), $1.05(6 \mathrm{H}, \mathrm{t}, J$ 7.1, C(2')H); ${ }^{13} \mathrm{C}\left\{{ }^{1} \mathrm{H}\right\}$ NMR (100 MHz, $\left.\mathrm{CDCl}_{3}\right) 192.3(\mathrm{CHO}), 154.7$ (C(2)), 134.4 (C(4)), $130.9(C(1)), 129.0(C(6)), 122.4(C(5)), 121.8$ $(C(3)), 49.0\left(C\left(1^{\prime}\right)\right), 12.4\left(C\left(2^{\prime}\right)\right)$; HRMS $\left(\mathrm{CI}^{+}\right) \mathrm{m} / \mathrm{z}$ : $[\mathrm{MH}]+$ Calcd for $\mathrm{C}_{11} \mathrm{H}_{16} \mathrm{NO}$ 178.1226; Found 178.1234. Experimental data is in agreement with reported values. ${ }^{56}$

\section{2-(Isopropyl(methyl)amino)benzaldehyde (21b)}

General procedure A (48 h) from 2-fluorobenzaldehyde (300 $\mu \mathrm{L}$, $4.75 \mathrm{mmol}), N$-isopropyl methylamine $(570 \mu \mathrm{L}, 5.46 \mathrm{mmol})$ and potassium carbonate $(755 \mathrm{mg}, 5.46 \mathrm{mmol})$ to give $\mathbf{2 1 b}(138 \mathrm{mg}$, $0.779 \mathrm{mmol}, 16 \%)$ as a dark orange oil. $\mathrm{R}_{\mathrm{f}}(20 \%$ hexane in DCM $)$ $0.51 ; v_{\max } / \mathrm{cm}^{-1} 1684,1595,1519,1454 ;{ }^{1} \mathrm{H}$ NMR $(400 \mathrm{MHz}$, $\left.\mathrm{CDCl}_{3}\right) 10.19(1 \mathrm{H}, \mathrm{s}, \mathrm{CHO}), 7.77(1 \mathrm{H}, \mathrm{dd}, J 7.7,1.8, \mathrm{C}(6) H), 7.45$ $(1 \mathrm{H}$, ddd, $J 8.2,7.2,1.8, \mathrm{C}(4) H), 7.07$ (1H, d, $J 8.1, \mathrm{C}(3) H), 7.00$ $(1 \mathrm{H}, \mathrm{t}, J 7.5, \mathrm{C}(5) H), 3.46\left(1 \mathrm{H}\right.$, hept, $\left.J 6.5, \mathrm{C}\left(2^{\prime}\right) H\right), 2.72(3 \mathrm{H}, \mathrm{s}$, $\left.\mathrm{C}\left(1^{\prime}\right) H_{3}\right), 1.14\left(6 \mathrm{H}, \mathrm{d}, J 6.6, \mathrm{C}\left(3^{\prime}\right) H_{3}\right) ;{ }^{13} \mathrm{C}\left\{{ }^{1} \mathrm{H}\right\} \mathrm{NMR}(100 \mathrm{MHz}$, $\left.\mathrm{CDCl}_{3}\right) 191.6(C \mathrm{HO}), 156.2(C(2)), 134.4(C(4)), 129.6(C(6))$, $128.9(C(1)), 121.3(C(5)), 120.3(C(3)), 59.1\left(C\left(2^{\prime}\right)\right), 32.4$ $\left(C\left(1^{\prime}\right)\right), 18.9\left(C\left(3^{\prime}\right)\right)$; HRMS (ESI+) m/z: $[\mathrm{MH}]+$ Calcd for $\mathrm{C}_{11} \mathrm{H}_{16} \mathrm{NO}$ 178.1226; Found 178.1226.

\section{2-(Benzyl(methyl)amino)benzaldehyde (21e)}

General procedure A $(48 \mathrm{~h})$ from 2-fluorobenzaldehyde $(1.0 \mathrm{~mL}$, $9.5 \mathrm{mmol}), N$-benzylmethylamine $(1.4 \mathrm{~mL}, 11 \mathrm{mmol})$ and potassium carbonate $(1.51 \mathrm{~g}, 10.9 \mathrm{mmol})$ to give $21 \mathrm{e}(246 \mathrm{mg}, 1.09$ mmol, $12 \%)$ as a yellow oil. $\mathrm{R}_{\mathrm{f}}(20 \%$ hexane in DCM) 0.68 ; $v_{\max } / \mathrm{cm}^{-1} 1712,1596,1484,1453 ;{ }^{1} \mathrm{H}$ NMR $\left(400 \mathrm{MHz}, \mathrm{CDCl}_{3}\right)$ $10.35(1 \mathrm{H}, \mathrm{s}, \mathrm{CHO}), 7.77(1 \mathrm{H}, \mathrm{dd}, J 7.7,1.7, \mathrm{C}(6) H), 7.47-7.38$ $(1 \mathrm{H}, \mathrm{m}, \mathrm{C}(4) H), 7.30-7.26\left(2 \mathrm{H}, \mathrm{m}, \mathrm{C}\left(5^{\prime}\right) H\right), 7.24-7.21(3 \mathrm{H}, \mathrm{m}$, $\left.\mathrm{C}\left(4^{\prime}\right) H+\mathrm{C}\left(6^{\prime}\right) H\right), 7.05(1 \mathrm{H}, \mathrm{d}, J 8.5, \mathrm{C}(3) H), 7.02(1 \mathrm{H}, \mathrm{t}, J 7.6$, $\mathrm{C}(5) H), 4.29\left(2 \mathrm{H}, \mathrm{s}, \mathrm{C}\left(2^{\prime}\right) H_{2}\right), 2.77\left(3 \mathrm{H}, \mathrm{s}, \mathrm{C}\left(1^{\prime}\right) H_{3}\right) ;{ }^{13} \mathrm{C}\left\{{ }^{1} \mathrm{H}\right\}$ NMR (100 MHz, $\left.\mathrm{CDCl}_{3}\right) 191.4(C \mathrm{HO}), 155.8(C(2)), 137.5$ $\left(C\left(3^{\prime}\right)\right), 134.8(C(4)), 130.3(C(6)), 128.6\left(C\left(5^{\prime}\right)\right), 128.2(C(1))$, $128.1\left(C\left(4^{\prime}\right)\right), 127.5\left(C\left(6^{\prime}\right)\right), 121.7(C(5)), 119.6(C(4)), 62.5$ $\left(C\left(2^{\prime}\right)\right), 42.4\left(C\left(1^{\prime}\right)\right) ; \mathrm{m} / z\left(\mathrm{ESI}^{+}\right) 226.0\left(\mathrm{MH}^{+}, 100 \%\right)$; HRMS $\left(\mathrm{ESI}^{+}\right) \mathrm{m} / \mathrm{z}:[\mathrm{MH}]+$ Calcd for $\mathrm{C}_{15} \mathrm{H}_{16} \mathrm{NO}$ 226.1226; Found 226.1226. Experimental data is in agreement with reported values. $^{57}$

\section{2-(Benzyl(ethyl)amino)benzaldehyde (21f)}

General procedure A $(48 \mathrm{~h})$ from 2-fluorobenzaldehyde $(1.0 \mathrm{~mL}$, $9.5 \mathrm{mmol}), N$-ethylbenzylamine $(1.6 \mathrm{~mL}, 11 \mathrm{mmol})$ and potassium carbonate $(1.51 \mathrm{~g}, 10.9 \mathrm{mmol})$ to give $21 \mathrm{f}(574 \mathrm{mg}, 2.40 \mathrm{mmol}$, $25 \%$ ) as a yellow oil, isolated as an inseparable mixture with aldehyde. $\mathrm{R}_{\mathrm{f}}\left(20 \%\right.$ hexane in DCM) $0.71 ; v_{\max } / \mathrm{cm}^{-1} 1739,1595$, 1481, 1453; ${ }^{1} \mathrm{H}$ NMR (400 MHz, $\left.\mathrm{CDCl}_{3}\right){ }^{1} \mathrm{H}$ NMR $(400 \mathrm{MHz}$, Chloroform- $d$ ) $\delta 10.50(1 \mathrm{H}, \mathrm{d}, J 0.5, \mathrm{CHO}), 7.84(1 \mathrm{H}, \mathrm{dd}, J 7.7$, 1.8, C(6)H)), 7.50 (1H, ddd, $J 8.2,7.2,1.8, \mathrm{C}(4) H), 7.35-7.25$ $\left(5 \mathrm{H}, \mathrm{m}, \mathrm{C}\left(5^{\prime}\right) H+\mathrm{C}\left(6^{\prime}\right) H+\mathrm{C}\left(7^{\prime}\right) H\right), 7.18(1 \mathrm{H}, \mathrm{d}, J 8.2, \mathrm{C}(3) H)$, $7.12(1 \mathrm{H}, \mathrm{t}, J 7.5, \mathrm{C}(5) H), 4.34\left(2 \mathrm{H}, \mathrm{s}, \mathrm{C}\left(3^{\prime}\right) H_{2}\right), 3.20(2 \mathrm{H}, \mathrm{q}, J 7.1$, $\left.\mathrm{C}\left(1^{\prime}\right) H_{2}\right), 1.09\left(3 \mathrm{H}, \mathrm{t}, J 7.1, \mathrm{C}\left(2^{\prime}\right) H_{3}\right) ;{ }^{13} \mathrm{C}\left\{{ }^{1} \mathrm{H}\right\}$ NMR $(100 \mathrm{MHz}$, $\left.\mathrm{CDCl}_{3}\right) 191.8(C \mathrm{HO}), 154.6(C(2)), 137.9\left(C\left(4^{\prime}\right)\right), 134.5(C(4))$, $130.4(C(1)), 129.2(C(6)), 128.5\left(C\left(5^{\prime}\right)\right.$ or $\left.C\left(6^{\prime}\right)\right), 128.4\left(C\left(5^{\prime}\right)\right.$ or $\left.C\left(6^{\prime}\right)\right), 127.4\left(C\left(7^{\prime}\right)\right), 122.7(C(5)), 122.1(C(3)), 58.5\left(C\left(3^{\prime}\right)\right)$, $49.7\left(C\left(1^{\prime}\right)\right), 11.9\left(C\left(2^{\prime}\right)\right) ; m / z\left(\mathrm{ESI}^{+}\right) 240.2\left(\mathrm{MH}^{+}, 100 \%\right)$; HRMS $\left(\mathrm{ESI}^{+}\right) \mathrm{m} / \mathrm{z}:[\mathrm{MH}]+$ Calcd for $\mathrm{C}_{16} \mathrm{H}_{18} \mathrm{NO}$ 240.1383; Found 240.1384. Experimental data is in agreement with reported values. $^{58}$

\section{2-(Dibenzylamino)benzaldehyde $(21 \mathrm{~h})$}

General procedure A $(24 \mathrm{~h})$ from 2-fluorobenzaldehyde $(500 \mu \mathrm{L}$, $4.75 \mathrm{mmol})$, dibenzylamine $(1.0 \mathrm{~mL}, 5.5 \mathrm{mmol})$ and potassium carbonate $(755 \mathrm{mg}, 5.46 \mathrm{mmol})$. A complex mixture was obtained, which was used in the following step (formation of $\mathbf{2 2} \mathbf{h}$ ) without further purification.

\section{2-(Diethylamino)-5-nitrobenzaldehyde (21i)}

General procedure A (24 h) from 2-fluoro-5-nitrobenzaldehyde (500 mg, $2.96 \mathrm{mmol})$, diethylamine $(350 \mu \mathrm{L}, 3.40 \mathrm{mmol})$ and potassium carbonate $(470 \mathrm{mg}, 3.40 \mathrm{mmol})$ to give $21 \mathrm{i}(326 \mathrm{mg}$, $1.47 \mathrm{mmol}, 50 \%)$ as a yellow solid. $\mathrm{R}_{\mathrm{f}}(20 \%$ hexane in DCM) 0.55 ; $v_{\max } / \mathrm{cm}^{-1} 1739,1592,1572,1435 ;{ }^{1} \mathrm{H}$ NMR $\left(400 \mathrm{MHz}, \mathrm{CDCl}_{3}\right)$ $10.01(1 \mathrm{H}, \mathrm{s}, \mathrm{CHO}), 8.59(1 \mathrm{H}, \mathrm{d}, J 2.8, \mathrm{C}(6) H), 8.20(1 \mathrm{H}, \mathrm{dd}, J$ 9.3, 2.9, C(4)H), $7.02(1 \mathrm{H}, \mathrm{d}, J 9.3, \mathrm{C}(3) H), 3.44(4 \mathrm{H}, \mathrm{q}, J$ 7.1, $\left.\mathrm{C}\left(1^{\prime}\right) H_{2}\right), 1.23\left(6 \mathrm{H}, \mathrm{t}, J 7.1, \mathrm{C}\left(2^{\prime}\right) H_{3}\right) ;{ }^{13} \mathrm{C}\left\{{ }^{1} \mathrm{H}\right\}$ NMR $(100 \mathrm{MHz}$, $\left.\mathrm{CDCl}_{3}\right) 188.5$ (CHO), $156.9(C(2)), 139.4(C(5)), 128.7(C(1))$, 128.5 (C(4)), $125.3(C(6)), 118.2(C(4)), 48.1$ (C(2')), 12.7 $\left(C\left(1^{\prime}\right)\right) ; \mathrm{m} / z\left(\mathrm{ESI}^{+}\right) 223.0\left(\mathrm{MH}^{+}, 100 \%\right) ; \mathrm{HRMS}\left(\mathrm{ESI}^{+}\right) \mathrm{m} / \mathrm{z}$ : $[\mathrm{MH}]+$ Calcd for $\mathrm{C}_{11} \mathrm{H}_{15} \mathrm{~N}_{2} \mathrm{O}_{3}$ 223.1077; Found 223.1079.

\section{5-Bromo-2-(diethylamino)benzaldehyde (21j)}

General procedure A $(24 \mathrm{~h})$ from 5-bromo-2-fluorobenzaldehyde (300 $\mu \mathrm{L}, 2.53 \mathrm{mmol})$, diethylamine $(300 \mu \mathrm{L}, 2.91 \mathrm{mmol})$ and potassium carbonate (402 $\mathrm{mg}, 2.91 \mathrm{mmol}$ ) to give $\mathbf{2 1 j}$ (304 mg, $1.19 \mathrm{mmol}, 47 \%)$ as a yellow oil. $\mathrm{R}_{\mathrm{f}}(20 \%$ hexane in DCM) 0.77 ; $v_{\max } / \mathrm{cm}^{-1} 1739,1680,1467,1585 ;{ }^{1} \mathrm{H}$ NMR $\left(400 \mathrm{MHz}, \mathrm{CDCl}_{3}\right)$ $10.24(1 \mathrm{H}, \mathrm{s}, \mathrm{CHO}), 7.89(1 \mathrm{H}, \mathrm{d}, J 2.6, \mathrm{C}(6) H), 7.56(1 \mathrm{H}, \mathrm{dd}, J$ 8.7, 2.6, C(4)H), $7.03(1 \mathrm{H}, \mathrm{d}, J 8.7, \mathrm{C}(3) H), 3.17$ (4H, q, $J 7.1$, $\left.\mathrm{C}\left(1^{\prime}\right) H_{2}\right), 1.05\left(6 \mathrm{H}, \mathrm{t}, J 7.1, \mathrm{C}\left(2^{\prime}\right) H_{3}\right) ;{ }^{13} \mathrm{C}\left\{{ }^{1} \mathrm{H}\right\} \mathrm{NMR}(100 \mathrm{MHz}$, $\left.\mathrm{CDCl}_{3}\right) 190.7(C \mathrm{HO}), 153.5(C(2)), 137.0(C(4)), 132.1(C(1))$, $131.7(C(6)), 123.7(C(3)), 115.6(C(5)), 49.1\left(C\left(1^{\prime}\right)\right), 12.4$ $\left(C\left(2^{\prime}\right)\right)$; HRMS $\left(\mathrm{CI}^{+}\right) \mathrm{m} / \mathrm{z}$ : $[\mathrm{M}]+$ Calcd for $\mathrm{C}_{11} \mathrm{H}_{15} \mathrm{BrNO} 256.0332$; Found 256.0333.

\section{Methyl (2'R,3R,6R,7aR)-3-(tert-butyl)-1'-ethyl-2'-methyl-5,7- dioxo-1',4'-dihydro-1H,2' $H, 3 H, 5 H$-spiro[pyrrolo[1,2- $c$ ]oxazole-6,3'-quinoline]-7a(7H)-carboxylate (22a)}

General procedure B $(6 \mathrm{~h})$ from tetramate $5 \mathbf{5}(100 \mathrm{mg}, 0.39 \mathrm{mmol})$ and 2-(diethylamino)benzaldehyde $21 \mathrm{a}(76 \mathrm{mg}, 0.43 \mathrm{mmol})$ to give spirocycle 22a (11 mg, $0.027 \mathrm{mmol}, 7 \%)$ via in vacuo concentration of reaction mixture instead of precipitation, isolated as a mixture of 2 diastereomers. $\mathrm{R}_{\mathrm{f}}\left(5 \% \mathrm{MeOH}\right.$ in EtOAc) $0.91 ;{ }^{1} \mathrm{H}$ NMR (600 MHz, $\mathrm{C}_{6} \mathrm{D}_{6}, 3: 1$ mixture of diastereomers) major isomer 22a", $7.13(1 \mathrm{H}, \mathrm{t}, J$ 7.3, C(11')H), $7.04(1 \mathrm{H}, \mathrm{d}, J 7.4$, $\left.\mathrm{C}\left(9^{\prime}\right) H\right), 6.77\left(1 \mathrm{H}, \mathrm{td}, J 7.4,1.1, \mathrm{C}\left(10^{\prime}\right) H\right), 6.57(1 \mathrm{H}, \mathrm{d}, J 8.2$, $\left.\mathrm{C}\left(12^{\prime}\right) H\right), 5.13(1 \mathrm{H}, \mathrm{s}, \mathrm{C}(3) H), 4.69\left(1 \mathrm{H}, \mathrm{d}, J 9.0, \mathrm{C}(1) H_{\mathrm{A}} \mathrm{H}_{\mathrm{B}}\right), 4.30$ $\left(1 \mathrm{H}, \mathrm{qd}, J 6.5,1.0, \mathrm{C}\left(5^{\prime}\right) H\right), 3.48\left(1 \mathrm{H}, \mathrm{d}, J 17.4, \mathrm{C}\left(7^{\prime}\right) H_{\mathrm{A}} \mathrm{H}_{\mathrm{B}}\right), 3.22$ $\left(3 \mathrm{H}, \mathrm{s}, \mathrm{CO}_{2} \mathrm{CH}_{3}\right), 3.19-3.10\left(1 \mathrm{H}, \mathrm{m}, \mathrm{C}\left(1^{\prime}\right) \mathrm{H}_{\mathrm{A}} \mathrm{H}_{\mathrm{B}}\right), 3.02(1 \mathrm{H}, \mathrm{d}, J$ 9.0, $\left.\mathrm{C}(1) \mathrm{H}_{\mathrm{A}} H_{\mathrm{B}}\right), 2.93\left(1 \mathrm{H}, \mathrm{dq}, J 14.5,7.2, \mathrm{C}\left(1^{\prime}\right) \mathrm{H}_{\mathrm{A}} H_{\mathrm{B}}\right), 2.50(1 \mathrm{H}$, 
d, $\left.J 17.1, \mathrm{C}\left(7^{\prime}\right) \mathrm{H}_{\mathrm{A}} H_{\mathrm{B}}\right), 1.10\left(3 \mathrm{H}, \mathrm{d}, J 6.6, \mathrm{C}\left(4^{\prime}\right) H_{3}\right), 1.03(9 \mathrm{H}, \mathrm{s}$, $\left.\mathrm{C}\left(\mathrm{CH}_{3}\right)_{3}\right), 0.90\left(3 \mathrm{H}, \mathrm{t}, J 7.1, \mathrm{C}\left(2^{\prime}\right) H_{3}\right) ;{ }^{13} \mathrm{C}\left\{{ }^{1} \mathrm{H}\right\} \mathrm{NMR}(125 \mathrm{MHz}$, $\left.\mathrm{C}_{6} \mathrm{D}_{6}\right)$ major isomer 22a", $199.5(C(7)), 177.8(C(5)), 168.1$ $\left(\mathrm{CO}_{2} \mathrm{CH}_{3}\right), 142.3\left(C\left(13^{\prime}\right)\right), 129.7\left(C\left(9^{\prime}\right)\right), 128.4\left(C\left(11^{\prime}\right)\right), 117.4$ $\left(C\left(8^{\prime}\right)\right), 117.2\left(C\left(10^{\prime}\right)\right), 112.2\left(C\left(12^{\prime}\right)\right), 98.9(C(3)), 78.6(C(7 \mathrm{a}))$, $69.7(C(1)), 56.4(C(6)), 56.2\left(C\left(5^{\prime}\right)\right), 52.9\left(\mathrm{CO}_{2} \mathrm{CH}_{3}\right), 45.6$ $\left(C\left(1^{\prime}\right)\right), 35.5\left(C\left(\mathrm{CH}_{3}\right)_{3}\right), 26.8\left(C\left(7^{\prime}\right)\right), 25.1\left(\mathrm{C}^{\prime}\left(\mathrm{CH}_{3}\right)_{3}\right), 15.3\left(C\left(4^{\prime}\right)\right)$, $13.9\left(C\left(2^{\prime}\right)\right) ; m / z\left(\mathrm{ESI}^{+}\right) 415.0\left(\mathrm{MH}^{+}, 100 \%\right)$; HRMS $\left(\mathrm{ESI}^{+}\right) \mathrm{m} / \mathrm{z}$ : $[\mathrm{MH}]+$ Calcd for $\mathrm{C}_{23} \mathrm{H}_{31} \mathrm{~N}_{2} \mathrm{O}_{5}$ 415.2227; Found 415.2225.

Methyl (3R,7aR)-1'-benzyl-3-(tert-butyl)-5,7-dioxo-2'-phenyl1',4' -dihydro-1H,2' $H, 3 H, 5 H$-spiro[pyrrolo[1,2-c] ]oxazole-6,3'quinoline]-7a(7H)-carboxylate $(22 \mathrm{~h})$

General procedure B $(6 \mathrm{~h})$ from tetramate $5 \mathbf{a}(50 \mathrm{mg}, 0.20 \mathrm{mmol})$

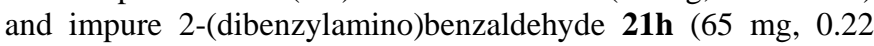
mmol) to give spirocycle $22 \mathrm{~h}$ (46 $\mathrm{mg}, 0.085 \mathrm{mmol}, 43 \%$ ) via in vacuo concentration of reaction mixture instead of precipitation, isolated as a mixture of 4 diastereomers. $\mathrm{R}_{\mathrm{f}}(25 \%$ EtOAc in petrol) $0.71 ;{ }^{1} \mathrm{H}$ NMR (400 MHz, $\mathrm{C}_{6} \mathrm{D}_{6}$, mixture of diastereomers) major isomer $7.36-7.29(2 \mathrm{H}, \mathrm{m}, \mathrm{Ar}), 7.18-6.91(10 \mathrm{H}, \mathrm{m}, \mathrm{Ar}), 6.78$ $\left(1 \mathrm{H}, \mathrm{t}, J 7.4, \mathrm{C}\left(10^{\prime}\right) H\right), 6.56\left(1 \mathrm{H}, \mathrm{d}, J 8.2, \mathrm{C}\left(12^{\prime}\right) H\right), 5.49(1 \mathrm{H}, \mathrm{s}$, $\left.\mathrm{C}\left(5^{\prime}\right) H\right), 5.06(1 \mathrm{H}, \mathrm{s}, \mathrm{C}(3) H), 4.74\left(1 \mathrm{H}, \mathrm{d}, J 9.0, \mathrm{C}(1) H_{\mathrm{A}} \mathrm{H}_{\mathrm{B}}\right), 4.39$ $\left(1 \mathrm{H}, \mathrm{d}, J 17.6, \mathrm{C}\left(1^{\prime}\right) H_{\mathrm{A}} \mathrm{H}_{\mathrm{B}}\right), 4.06\left(1 \mathrm{H}, \mathrm{d}, J 17.6, \mathrm{C}\left(1^{\prime}\right) \mathrm{H}_{\mathrm{A}} H_{\mathrm{B}}\right), 3.53$ $\left(1 \mathrm{H}, \mathrm{d}, J 17.2, \mathrm{C}\left(7^{\prime}\right) H_{\mathrm{A}} \mathrm{H}_{\mathrm{B}}\right), 3.10\left(1 \mathrm{H}, \mathrm{d}, J 8.6, \mathrm{C}(1) \mathrm{H}_{\mathrm{A}} H_{\mathrm{B}}\right), 2.94$ $\left(3 \mathrm{H}, \mathrm{s}, \mathrm{CO}_{2} \mathrm{CH}_{3}\right), 2.62\left(1 \mathrm{H}, \mathrm{d}, J 17.2, \mathrm{C}\left(7^{\prime}\right) \mathrm{H}_{\mathrm{A}} H_{\mathrm{B}}\right), 1.02(9 \mathrm{H}, \mathrm{s}$, $\left.\mathrm{C}\left(\mathrm{CH}_{3}\right)_{3}\right) ;{ }^{13} \mathrm{C}\left\{{ }^{1} \mathrm{H}\right\}$ NMR $\left(100 \mathrm{MHz}, \mathrm{C}_{6} \mathrm{D}_{6}\right)$ major isomer 199.4 $(C(7)), 175.4(C(5)), 167.5\left(C_{2} \mathrm{CH}_{3}\right), 144.7(\mathrm{Ar}), 138.6(\mathrm{Ar})$, 129.6 (C(9')), 128.8 - 127.1 (Ar), $126.5(\mathrm{Ar}), 117.6\left(C\left(8^{\prime}\right)\right), 111.0$ (C(12')), $98.8(C(3)), 79.0(C(7 \mathrm{a})), 70.0(C(1)), 64.0\left(C\left(5^{\prime}\right)\right), 57.8$ $(C(6)), 53.8\left(C\left(1^{\prime}\right)\right), 53.0\left(\mathrm{CO}_{2} \mathrm{CH}_{3}\right), 35.6\left(C\left(\mathrm{CH}_{3}\right)_{3}\right), 27.0\left(C\left(7^{\prime}\right)\right)$, $25.2\left(\mathrm{C}\left(\mathrm{CH}_{3}\right)_{3}\right) ; \mathrm{m} / \mathrm{z}\left(\mathrm{ESI}^{+}\right) 539.2\left(\mathrm{MH}^{+}, 100 \%\right) ; \mathrm{HRMS}\left(\mathrm{ESI}^{+}\right)$ $\mathrm{m} / \mathrm{z}:[\mathrm{MH}]+$ Calcd for $\mathrm{C}_{33} \mathrm{H}_{35} \mathrm{~N}_{2} \mathrm{O}_{5}$ 539.2540; Found 539.2537.

\section{5-Bromo-2-(pyrrolidin-1-yl)benzaldehyde (23)}

General procedure A $(6 \mathrm{~h})$ from 5-bromo-2-fluorobenzaldehyde (300 $\mu \mathrm{L}, 2.53 \mathrm{mmol})$, pyrrolidine $(250 \mu \mathrm{L}, 3.04 \mathrm{mmol})$ and potassium carbonate $(420 \mathrm{mg}, 3.04 \mathrm{mmol})$ to give $23(478 \mathrm{mg}$, $1.88 \mathrm{mmol}, 74 \%)$ as a brown oil. $\mathrm{R}_{\mathrm{f}}(5 \%$ EtOAc in petrol) 0.26 ; $v_{\max } / \mathrm{cm}^{-1} 1739,1559,1458,1152 ;{ }^{1} \mathrm{H}$ NMR $\left(400 \mathrm{MHz}, \mathrm{CDCl}_{3}\right)$ $10.01(1 \mathrm{H}, \mathrm{s}, \mathrm{CHO}), 7.78(1 \mathrm{H}, \mathrm{d}, J 2.5, \mathrm{C}(6) H), 7.41(1 \mathrm{H}, \mathrm{dd}, J$ 9.0, 2.6, C(4)H), $6.71(1 \mathrm{H}, \mathrm{d}, J 9.0, \mathrm{C}(3) H), 3.38-3.29(4 \mathrm{H}, \mathrm{m}$, $\left.\mathrm{C}\left(4^{\prime}\right) H_{2}\right), 2.02-1.96\left(4 \mathrm{H}, \mathrm{m}, \mathrm{C}\left(3^{\prime}\right) H_{2}\right) ;{ }^{13} \mathrm{C}\left\{{ }^{1} \mathrm{H}\right\} \mathrm{NMR}(100 \mathrm{MHz}$, $\left.\mathrm{CDCl}_{3}\right) 188.9$ (CHO), $149.0(C(2)), 136.8(C(4)), 134.7(C(6))$, $124.0(C(1)), 116.6(C(3)), 108.3(C(5)), 53.0\left(C\left(2^{\prime}\right)\right), 26.1$ $\left(C\left(1^{\prime}\right)\right) ; m / z\left(\mathrm{ESI}^{+}\right) 254.0\left(\mathrm{MH}^{+}, 100 \%\right) ; \mathrm{HRMS}\left(\mathrm{ESI}^{+}\right) \mathrm{m} / \mathrm{z}$ : $[\mathrm{MH}]+$ Calcd for $\mathrm{C}_{11} \mathrm{H}_{12} \mathrm{BrNO} 254.0175$; Found 254.0175.

\section{4-(Pyrrolidin-1-yl)-[1,1'-biphenyl]-3-carbaldehyde (24)}

5-bromo-2-(pyrrolidin-1-yl)benzaldehyde (430 mg, $1.68 \mathrm{mmol}$ ) and phenylboronic acid $(310 \mathrm{mg}, 2.52 \mathrm{mmol})$ were dissolved in anhydrous THF $(20 \mathrm{~mL})$. To the solution were added $\mathrm{Pd}(\mathrm{dppf}) \mathrm{Cl}_{2}$ (62.8 mg, $0.216 \mathrm{mmol})$ and $\mathrm{Cs}_{2} \mathrm{CO}_{3}\left(1.65 \mathrm{~g}\right.$ in $2 \mathrm{~mL} \mathrm{H} \mathrm{H}_{2} \mathrm{O}, 5.07$ $\mathrm{mmol}$ ). The mixture was stirred at room temperature for $40 \mathrm{~min}$, and then heated to reflux for $48 \mathrm{~h}$. The mixture was then allowed to cool to room temperature, diluted with EtOAc, filtered through Celite and concentrated. The residue was taken up into EtOAc, washed with brine and saturated aqueous $\mathrm{NaHCO}_{3}(2 \times 15 \mathrm{~mL})$ and concentrated in vacuo, and then purified by flash column chromatography to give biphenyl 24 (267 mg, $1.06 \mathrm{mmol}, 63 \%)$ as a yellow oil. $\mathrm{R}_{\mathrm{f}}\left(5 \%\right.$ EtOAc in petrol) $0.77 ; v_{\max } / \mathrm{cm}^{-1} 1738$, 1541, 1494, 1449; ${ }^{1} \mathrm{H}$ NMR (400 MHz, $\left.\mathrm{CDCl}_{3}\right) 10.17(1 \mathrm{H}, \mathrm{s}$, CHO), $7.96(1 \mathrm{H}, \mathrm{d}, J 2.4, \mathrm{C}(3) H), 7.67(1 \mathrm{H}, \mathrm{dd}, J 8.8,2.4, \mathrm{C}(6) H)$, $7.62-7.56\left(2 \mathrm{H}, \mathrm{m}, \mathrm{C}\left(5^{\prime}\right) H\right), 7.47-7.38\left(2 \mathrm{H}, \mathrm{m}, \mathrm{C}\left(6^{\prime}\right) H\right), 7.35-$ $7.27\left(1 \mathrm{H}, \mathrm{m}, \mathrm{C}\left(7^{\prime}\right) H\right), 6.92(1 \mathrm{H}, \mathrm{d}, J 8.8, \mathrm{C}(5) H), 3.47-3.37(4 \mathrm{H}$, m, C $\left.\left(2^{\prime}\right) H\right), 2.07-1.97\left(4 \mathrm{H}, \mathrm{m}, \mathrm{C}\left(3^{\prime}\right) H\right) ;{ }^{13} \mathrm{C}\left\{{ }^{1} \mathrm{H}\right\}$ NMR $(100$
$\left.\mathrm{MHz}, \mathrm{CDCl}_{3}\right) 190.3$ (CHO), $149.4(C(3)), 140.0$ (C(4')), 132.9 $(C(6)), 131.3(C(3)), 129.4(C(2)), 128.9\left(C\left(6^{\prime}\right)\right), 126.7\left(C\left(7^{\prime}\right)\right)$, $126.3\left(C\left(5^{\prime}\right)\right), 123.1(C(4)), 115.3(C(5)), 53.0$ (C(2')), 26.1 $\left(C\left(1^{\prime}\right)\right)$; HRMS $\left(\mathrm{SI}^{+}\right) \mathrm{m} / \mathrm{z}$ : $[\mathrm{M}]+$ Calcd for $\mathrm{C}_{17} \mathrm{H}_{17} \mathrm{NO} 251.1310$; Found 251.1298.

\section{Supporting Information}

The Supporting Information is available free of charge on the ACS Publications website at DOI: 10.1021/acs.joc.xxxx. ${ }^{1} \mathrm{H}$ and ${ }^{13} \mathrm{C}$ NMR spectra; calculated energies; X-ray crystallographic data (PDF).

\section{Acknowledgements}

The research leading to these results has received funding from the People Programme (Marie Curie Actions) of the European Union's Seventh Framework Programme (FP7/2007-2013) under REA Grant Agreement $n^{\circ} 316955$. We would also like to thank Remi Tirfoin for collection of some of the X-ray diffraction data.

\section{References}

(1) Saleem, M.; Nazir, M.; Ali, M. S.; Hussain, H.; Lee, Y. S.; Riaz, N.; Jabbar, A. Antimicrobial Natural Products: An Update on Future Antibioticdrug Candidates. Nat. Prod. Rep. 2010, 27 (2), 238-254.

(2) Boucher, H. W.; Talbot, G. H.; Benjamin, D. K.; Bradley, J.; Guidos, R. J.; Jones, R. N.; Murray, B. E.; Bonomo, R. A.; Gilbert, D. 10 x '20 Progress--Development of New Drugs Active Against Gram-Negative Bacilli: An Update From the Infectious Diseases Society of America. Clin. Infect. Dis. 2013, 56 (12), 1685-1694.

(3) Butler, M. S.; Blaskovich, M. A.; Cooper, M. A. Antibiotics in the Clinical Pipeline at the End of 2015. J. Antibiot. 2017, 70 (1), 3-24.

(4) Christoffersen, R. E. Antibiotics - An Investment Worth Making? Nat. Biotechnol. 2006, 24 (12), 1512-1514.

(5) Fox, J. L. The Business of Developing Antibacterials. Nat. Biotechnol. 2006, 24 (12), 1521-1528.

(6) Katz, M. L.; Mueller, L. V; Polyakov, M.; Weinstock, S. F. Where Have All the Antibiotic Patents Gone? Nat. Biotechnol. 2006, 24 (12), 1529-1531.

(7) Kraus, C. N. Low Hanging Fruit in Infectious Disease Drug Development. Curr. Opin. Microbiol. 2008, 11 (5), 434438.

(8) Kinch, M. S.; Patridge, E.; Plummer, M.; Hoyer, D. An Analysis of FDA-Approved Drugs for Infectious Disease: Antibacterial Agents. Drug Discov. Today 2014, 19 (9), 1283-1287.

(9) Anderson, A. C.; Pollastri, M. P.; Schiffer, C. A.; Peet, N. P. The Challenge of Developing Robust Drugs to Overcome Resistance. Drug Discov. Today 2011, 16 (17-18), 755761.

(10) Hurdle, J. G.; O’Neill, A. J.; Chopra, I.; Lee, R. E. Targeting Bacterial Membrane Function: An Underexploited Mechanism for Treating Persistent Infections. Nat. Rev. Microbiol. 2011, 9 (1), 62-75.

(11) Wright, G. D. Molecular Mechanisms of Antibiotic Resistance. Chem. Commun. 2011, 47 (14), 4055.

(12) Brown, D. G.; Lister, T.; May-Dracka, T. L. New Natural Products as New Leads for Antibacterial Drug Discovery. Bioorg. Med. Chem. Lett. 2014, 24, 413-418. 
(13) Walsh, C. T.; Wencewicz, T. A. Prospects for New Antibiotics: A Molecule-Centered Perspective. J. Antibiot. 2014, 67 (1), 7-22.

(14) Butera, J. A. Phenotypic Screening as a Strategic Component of Drug Discovery Programs Targeting Novel Antiparasitic and Antimycobacterial Agents: An Editorial. J. Med. Chem. 2013, 56 (20), 7715-7718.

(15) Zheng, W.; Thorne, N.; McKew, J. C. Phenotypic Screens as a Renewed Approach for Drug Discovery. Drug Discov. Today 2013, 18 (21-22), 1067-1073.

(16) Newman, D. J.; Cragg, G. M. Natural Products as Sources of New Drugs over the 30 Years from 1981 to 2010. J. Nat. Prod. 2012, 75, 311-335.

(17) Hughes, J. P.; Rees, S. S.; Kalindjian, S. B.; Philpott, K. L. Principles of Early Drug Discovery. Br. J. Pharmacol. 2011, 162 (6), 1239-1249.

(18) Swinney, D. C.; Anthony, J. How Were New Medicines Discovered? Nat. Rev. Drug Discov. 2011, 10 (7), 507-519.

(19) Miller, A. A.; Bundy, G. L.; Mott, J. E.; Skepner, J. E.; Boyle, T. P.; Harris, D. W.; Hromockyj, A. E.; Marotti, K. R.; Zurenko, G. E.; Munzner, J. B.; et al. Discovery and Characterization of QPT-1, the Progenitor of a New Class of Bacterial Topoisomerase Inhibitors. Antimicrob. Agents Chemother. 2008, 52 (8), 2806-2812.

(20) Basarab, G. S.; Dumas, J.; Hill, P. WO2009004382 A2, 2009.

(21) Basarab, G. S.; Galullo, V.; Degrace, N.; Hauck, S.; Joubran, C.; Wesolowski, S. S. Synthesis of a Tetrahydronaphthyridine Spiropyrimidinetrione Dna Gyrase Inhibiting Antibacterial Agent - Differential Substitution at All Five Carbon Atoms of Pyridine. Org. Lett. 2014, 16 (24), 6456-6459.

(22) Basarab, G. S.; Brassil, P.; Doig, P.; Galullo, V.; Haimes, H. B.; Kern, G.; Kutschke, A.; McNulty, J.; Schuck, V. J. A.; Stone, G.; et al. Novel DNA Gyrase Inhibiting Spiropyrimidinetriones with a Benzisoxazole Scaffold: Sar and in Vivo Characterization. J. Med. Chem. 2014, 57 (21), 9078-9095.

(23) Huband, M. D.; Bradford, P. A.; Otterson, L. G.; Basarab, G. S.; Kutschke, A. C.; Giacobbe, R. A.; Patey, S. A.; Alm, R. A.; Johnstone, M. R.; Potter, M. E.; et al. In Vitro Antibacterial Activity of AZD0914, a New Spiropyrimidinetrione DNA Gyrase/Topoisomerase Inhibitor with Potent Activity against Gram-Positive, Fastidious Gram-Negative, and Atypical Bacteria. Antimicrob. Agents Chemother. 2015, 59 (1), 467-474.

(24) Basarab, G. S.; Kern, G. H.; McNulty, J.; Mueller, J. P.; Lawrence, K.; Vishwanathan, K.; Alm, R. A.; Barvian, K.; Doig, P.; Galullo, V.; et al. Responding to the Challenge of Untreatable Gonorrhea: ETX0914, a First-in-Class Agent with a Distinct Mechanism-of-Action against Bacterial Type II Topoisomerases. Sci. Rep. 2015, 5 (July), 1-14.

(25) Kern, G.; Palmer, T.; Ehmann, D. E.; Shapiro, A. B.; Andrews, B.; Basarab, G. S.; Doig, P.; Fan, J.; Gao, N.; Mills, S. D.; et al. Inhibition of Neisseria Gonorrhoeae Type II Topoisomerases by the Novel Spiropyrimidinetrione AZD0914. J. Biol. Chem. 2015, 290 (34), 20984-20994.

(26) Meth-Cohn, O.; Suschitzky, H. Heterocycles by Ring Closure of Ortho-Substituted t-Anilines (The t-Amino Effect). Adv. Heterocycl. Chem. 1972, 14, 211-278.

(27) Verboom, W.; Reinhoudt, D. N.; Visser, R.; Harkema, S. "Tert-Amino Effect" in Heterocyclic Synthesis. Formation of N-Heterocycles by Ring-Closure Reactions of Substituted 2-Vinyl-N,N-Dialky Lanilines. J. Org. Chem. 1984, 49 (2), 269-276.

Nijhuis, W. H. N.; Verboom, W.; El-fadl, A. A.; Harkema, S.; Reinhoudt, D. N. Stereochemical Aspects of the "TertAmino Effect". 1. Regioselectivity in the Synthesis of Pyrrolo[1,2-a]Quinolines and Benzo[c]Quinolizines. J. Org. Chem. 1989, 54 (1), 199-209.

(29) Ryabukhin, S. V; Plaskon, A. S.; Volochnyuk, D. M.; Shivanyuk, A. N.; Tolmachev, A. A. A One-Pot Fusion of Nitrogen-Containing Heterocycles. Synthesis 2007, No. 18, 2872-2886.

(30) Rabong, C.; Hametner, C.; Mereiter, K.; Kartsev, V. G.; Jordis, U. Scope and Limitations of the T-Reaction Employing Some Functionalized C-H-Acids and Naturally Occurring Secondary Amines. Heterocycles 2008, 75 (4), 799-838.

(31) Ruble, J. C.; Hurd, A. R.; Johnson, T. A.; Sherry, D. A.; Barbachyn, M. R.; Toogood, P. L.; Bundy, G. L.; Graber, D. R.; Kamilar, G. M. Synthesis of (-)-PNU-286607 by Asymmetric Cyclization of Alkylidene Barbiturates. J. Am. Chem. Soc. 2009, 131 (11), 3991-3997.

(32) Deeva, E. V.; Glukhareva, T. V.; Zybina, N. A.; Morzherin, Y. Y. Stereoselective Synthesis of Spiro Derivatives of 2,4Dimethyl-2,3,4,4a,5,6-Hexahydro-6H-Benzo[c]Quinolizine. Russ. Chem. Bull. Int. Ed. 2005, 54 (6), 1537-1538.

(33) Krasnov, K. A.; Kartsev, V. G. Chemical Modification of Plant Alkaloids. 6. T-Reactions of Anabasine Derivatives. Chem. Nat. Compd. 2011, 46 (6), 915-919.

(34) Yong-Chul, J.; Moloney, M. G. Equisetin, Reutericyclin and Streptolodygin as Natural Product Lead Structures for Novel Antibiotic Libraries. Future Med. Chem. 2015, 7 (14), 1861-1877.

(35) Anwar, M.; Moloney, M. G. Chiral Bicyclic Tetramates as Non-Planar Templates for Chemical Library Synthesis. Chem. Biol. Drug Des. 2013, 81, 645-649.

(36) Butt, N. A.; Moody, C. J. Synthesis of Spirotetramates via a Diels-Alder Approach. Org. Lett. 2011, 13 (9), 2224-2227.

(37) Fischer, R.; Pontzen, R.; Vermeer, R.; Franken, E.-M.; Malsam, O.; Pitta, L.; Reckmann, U. 4'4'-DioxaspiroSpirocyclically Substituted Tetramates, 2011.

(38) Ramakumar, K.; Maji, T.; Partridge, J. J.; Tunge, J. A. Synthesis of Spirooxindoles via the Tert-Amino Effect. Org. Lett. 2017, 19 (15), 4014-4017.

(39) Kempf, K.; Kempf, O.; Orozco, M.; Bilitewski, U.; Schobert, R. Synthesis and Structural Revision of the Fungal Tetramic Acid Metabolite Spiroscytalin. J. Org. Chem. 2017, 82 (15), 7791-7795.

(40) Josa-Culleré, L.; Moloney, M. G.; Thompson, A. L. Stereoselectivity in the Reduction of Bicyclic Tetramates. Synlett 2016, 27 (11), 1677-1681.

D’yachenko, E. V.; Glukhareva, T. V.; Nikolaenko, E. F.; Tkachev, A. V.; Morzherin, Y. Y. Tert-Amino Effect in Heterocyclic Chemistry. Synthesis of Hydrogenated Spiro Derivatives of Quinolines. Russ. Chem. Bull. 2004, 53 (6), $1240-1247$.

Krasnov, K. A.; Kartsev, V. G. Synthesis of Spiroheterocyclic Systems from Barbituric Acids and N,NDisubstituted o-Aminobenzaldehydes. Russ. J. Org. Chem. 2005, 41 (6), 901-906.

Platonova, A. Y.; Deeva, E. V.; Zimovets, O. A.; 
Shatunova, D. V.; El'tsov, O. S.; Slepukhin, P. A.; Glukhareva, T. V.; Morzherin, Y. Y. Regioselective Reaction of Ortho-Piperidinobenzaldehydes with Pyrazolone. Russ. Chem. Bull. Int. Ed. 2011, 60 (5), 961964.

(44) Andrews, M. D.; Brewster, A. G.; Moloney, M. G. Diastereocontrolled Synthesis of Hydroxylated Lactams. $J$. Chem. Soc. Perkin Trans. 1 2002, 80-90.

(45) Krasnov, K. A.; Kartsev, V. G.; Khrustalev, V. N. Diastereoselective Synthesis of 1-Alkyl-2,4,6Trioxoperhydropyrimidine-5- Spiro-3'-(1',2',3',4'Tetrahydroquinolines). Tetrahedron 2010, 66 (32), 60546061 .

(46) Saurí, J.; Parella, T.; Espinosa, J. F. CLIP-HSQMBC: Easy Measurement of Small Proton-Carbon Coupling Constants in Organic Molecules. Org. Biomol. Chem. 2013, 11 (27), 4473.

(47) Karplus, M. Contact Electron-Spin Coupling of Nuclear Magnetic Moments Contact Electron-Spin Coupling of Nuclear Magnetic Moments. J. Chem. Phys. 1959, 30 (1), $10-15$.

(48) Single crystal X-ray diffraction data were collected using a (Rigaku) Supernova A diffractometer fitted with an Oxford CryoSystems CryoStream open flow cooling device. ${ }^{59}$ Raw frame data were collected and reduced using CrysAlisPro; the structures were solved and refined using SuperFlip ${ }^{60}$ within the CRYSTALS refinement software suite. ${ }^{61}$ Hydrogen atoms were generally visible in the difference Fourier map and refined with restraints prior to inclusion in the final refinement using a riding model. ${ }^{62}$ In each case, the Flack x parameter ${ }^{63}$ was determined by inclusion in the model and refinement using full-matrix least-squares. ${ }^{64}$ Selected refinement details for each structure are given below and full details can be found in the ESI (CIF). Crystallographic data have also been deposited with the Cambridge Crystallographic Data Centre (CCDC 19171091917113) and copies of these data can be obtained free of charge via http://www.ccdc.cam.ac.uk/data_request/cif.

(49) Anwar, M.; Moloney, M. G. Efficient Enantioselective Synthesis of Tetramic Acids and Lactams from Threonine. Tetrahedron Lett. 2007, 48 (41), 7259-7262.

(50) Panduwawala, T. D.; Iqbal, S.; Tirfoin, R.; Moloney, M. G. Chemoselectivity and Stereoselectivity of Cyclisation Pathways Leading to Bicyclic Tetramates Controlled by Ring-Chain Tautomerisation in Thiazolidines. Org. Biomol. Chem. 2016, 14 (19), 4464-4478.

(51) Andrews, M. D.; Brewster, A. G.; Crapnell, K. M.; Ibbett, A. J.; Jones, T.; Moloney, M. G.; Prout, K.; Watkin, D. Regioselective Dieckmann Cyclisations Leading to Enantiopure Highly Functionalised Tetramic Acid Derivatives. J. Chem. Soc. Perkin Trans. 1 1998, 223-235.

(52) Jeong, Y.-C.; Anwar, M.; Bikadi, Z.; Hazai, E.; Moloney, M. G. Natural Product Inspired Antibacterial Tetramic Acid Libraries with Dual Enzyme Inhibition. Chem. Sci. 2013, 4, 1008-1015.

(53) Krogsgaard-Larsen, N.; Begtrup, M.; Herth, M. M. Novel, Versatile Three-Step Synthesis of 1,2,3,4,10,10aHexahydro- Pyrazino[1,2-a]Indoles by Intramolecular Carbene-Mediated C-H Insertion. Synthesis 2010, No. 24, 4287-4299.

(54) Barbachyn, M. R.; Dobrowolski, P. J.; Hurd, A. R.; Mcnnamara, D. J.; Palmer, J. R.; Romero, A. G.; Ruble, J. C.; Sherry, D. A.; Thomasco, P. L. WO2004031195 A1, 2004.
On careful inspection of the raw frame images, weak additional reflections were visible. These were indexed with respect to the main unit cell reflections using the vector $0.0015(6) 00.4348(6)$. The reflections were too weak to integrate and use for refinement so the structure reported is the three dimensional, average structure.

(56) Chen, Z.; Liu, J.; Pei, H.; Liu, W.; Chen, Y.; Wu, J.; Li, W.; Li, Y. Directed Amination of Aryl Methyl Ethers Mediated by $\mathrm{Ti}(\mathrm{NMe} 2) 4$ at Room Temperature. Org. Lett. 2015, 17 (14), 3406-3409.

(57) Murarka, S.; Zhang, C.; Konieczynska, M. D.; Seidel, D. Lewis Acid Catalyzed Formation of Tetrahydroquinolines via an Intramolecular Redox Process. Org. Lett. 2009, 11 (1), 129-132.

(58) Sun, H.; Yang, C.; Gao, F.; Li, Z.; Xia, W. Oxidative C-C Bond Cleavage of Aldehydes via Visible-Light Photoredox Catalysis. Org. Lett. 2013, 15 (3), 624-627.

(59) Cosier, J.; Glazer, A. M. A Nitrogen-Gas-Stream Cryostat for General X-ray Diffraction Studies. J. Appl. Cryst. 1986, 19, 105-107.

(60) Palatinus, L.; Chapuis. G. SUPERFLIP - a computer program for the solution of crystal structures by charge flipping in arbitrary dimensions. J. Appl. Cryst., 2007, 40, 786-790.

(61) Betteridge, P.W.; Carruthers, J.R.; Cooper, R.I.; Prout, K.; Watkin, D.J. CRYSTALS version 12: software for guided crystal structure analysis. J. Appl. Cryst. 2003, 36, 1487; Parois, P.; Cooper, R. I.; Thompson, A. L. Crystal structures of increasingly large molecules: meeting the challenges with CRYSTALS software Chem. Cent. J., 2015 9:30.

(62) Cooper, R.I.; Thompson, A.L.; Watkin, D.J. CRYSTALS Enhancements: Dealing with Hydrogen Atoms in Refinement. J. Appl. Cryst. 2010, 43, 1100-1107.

(63) Flack, H. D. On Enantiomorph-Polarity Estimation. Acta Cryst. 1983 A39, 876-881; Flack, H. D.; Bernardinelli, G. Reporting and evaluating absolute-structure and absoluteconfiguration determinations. J. Appl. Cryst. 2000, 33, 1143-1148.

(64) Thompson, A. L.; Watkin, D. J. CRYSTALS enhancements: absolute structure determination. J. Appl. Cryst. 2011, 44, 1017-1022. 